\title{
Three-dimensional dynamics of functionally graded piezoelectric cylindrical panels by a semi-analytical approach
}

Xu Liang ${ }^{1}$, Yu Deng ${ }^{1}$, Zeng $\mathrm{Cao}^{1}$, Xue Jiang ${ }^{2, *}$, Titao Wang ${ }^{1}$, Yongdu Ruan ${ }^{1}$, Xing Zha ${ }^{1}$

${ }^{1}$ Dept. of Ocean Engineering, Zhejiang Univ., 866 Yuhangtang Rd., Hangzhou, Zhejiang Province, 310058, P. R. China.

${ }^{2}$ Department of naval architecture, ocean and marine engineering, University of Strathclyde, G15AE, UK.

* Corresponding author

Email: xue.jiang@strath.ac.uk 


\section{Abstract:}

Nowadays, intelligent piezoelectric materials are widely used in transducer devices and aerospace structures. To investigate the dynamic behaviour of a functionally graded piezoelectric material (FGPM) cylindrical panel, a semi-analytical approach by introducing the Laplace transform, differential quadrature method, state space approach and Durbin's numerical inversion of Laplace transform is presented. The accuracy of this method is validated by comparing the results with the published literature and by ANSYS under various boundary conditions. Convergence studies showed the proposed approach has a quick convergence rate with growing sample point numbers and increasing layer numbers. The analysis of initial electric potential on the outer surface and bottom surface indicated that when the plus and minus signs of two sides are opposite, the value of central electric potential will decrease. Under simply supported condition, if FG index $\gamma$ increases, the deflection amplitude of the panel will increase, while the electric potential amplitude decreases. Moreover, the amplitude of displacements along the radial direction becomes larger when the central angle increases, but the period time decreases at the same time. The proposed method fills a gap of 3-D semi-analytical investigations for the dynamic behaviour of FGPM cylindrical panels in different boundary conditions.

Keywords: functionally graded piezoelectric cylindrical panels; differential quadrature method; state space approach; numerical inversion of Laplace transform; transient response. 


\section{Introduction}

Functionally graded materials (FGMs) have gained considerable attentions nowadays [1-3]. They play very important roles in the aerospace industry and energy conversion systems. The advantageous materials use advanced material compounding technology to make the elements changing continuously in gradient without stress concentration and obvious surface. So the behaviour and function of the structure can also change in gradient. As for piezoelectric materials, they are widely introduced in intelligent structures because of their direct and converse piezoelectric effects, especially in terms of material damage self-diagnosis, vibration damping and noise control [4-7]. But the fact that stress concentration will shorten the lifetime of piezoelectric devices has annoyed many scientists. In particular, the high temperature environment in which the space shuttle flies and the energy conversion during satellite orbit operation. So as to break those limitations of conventional piezoelectric material, a superior composite material named functionally graded piezoelectric materials (FGPMs) has been developed [8-10].

In recent years, more and more researchers have investigated the behaviours of FGPM structures [11-13]. Lian [14] analysed the response of functionally graded piezoelectric cantilever beam by the use of multi-parameter perturbation method and made clear how the piezoelectric effects influence the behaviour. Salahaldin et al. [15] investigated the free vibration of a thick FGP plate based on the three dimensional (3-D) Element-Free Galerkin (EFG) method. In his paper, 3D moving least squares (MLS) approximation was applied to get the shape functions. Under simply supported conditions, Jandaghian et al. [16] used Eringen's Kirchhoff plate theory to describe the static response of FGPMs nanoscale plates. Wu et al. [17] proposed the free vibration response of simply supported FGP shells based on the high order theory. To obtain the fundamental frequencies, 
three charge equations depended by driving signal of piezoelectrics had been given. In Mallek's [18] work, static behaviour of an FGPM shell has been investigated by third-order shear deformation theory and finite element method (FEM). The effect analysis of electric potential on the response of an FGP hollow sphere was carried out using the Tresca yield criterion by Ali et al. [19]. The above researches, however, only analyse the statics or free vibration of FGPM structures without transient dynamics being involved in.

To obtain the transient responses of FGPM structures, many groups have done a lot of work. Using Timoshenko beam theory, Zhu et al. [20] investigated the free vibration and dynamic behaviour of an FGP curved beam. Under a moving heat source, Ma et al. [21] studied thermopiezoelectric dynamics of an FGP rod based on fractional order theory. Laplace transform and numerical Laplace inversion were also introduced to solve the problem. Based on the first shear deformation theory (FSDT), Zhu et al. [22] proposed a unified method for static behaviour and dynamic response of FGP rectangular plates with different boundaries. On the basis of Laplace transform and its inversion method, Bodaghi et al. [23] established an analytical approach to analyse the statics and dynamics of an FGP cylindrical panel with simply supported edges. In his paper, the governing equations were derived by FSDT and Hamilton's principle. Similarly, for Hamilton's principle, Mehralian et al. [24] presented an analysis of transient vibration of a bimorph FGP cylindrical nanoshell with nonlocal strain gradient theory. As mentioned above, those methods are relatively single and some theory assumptions may cause inevitable errors when ignoring some variables.

The advantages of state space method (SSM) are that all the fundamental equations of 3-D elasticity are exactly satisfied and all nine elastic variables are taken into account [25]. Due to the 
advantageous features compared with other theories, SSM has been efficiently introduced to deal with the static problems of FGPM structures. To investigate the free vibration of an FGPM beam, Li et al. [26] extended state-space based differential quadrature method (SSDQM) under different boundary conditions. Similarly, using the SSDQM, the natural frequencies analysis of FGP annular plates had been carried out in Jodaei's [27] work. In the work, comparative behaviour modeling by an artificial neural network (ANN) was used to solve the problem with different conditions of edges.

Based on the SSM, Chen et al. [28] studied the static behaviour of an FGP hollow cylinder with internal fluid. The arbitrary thick of cylinder and non-viscous compressible fluid medium were taken into consideration. However, due to the complexity of three dimensional FGPM structures, the transient vibration has not been investigated yet by SSM.

In this paper, a semi-analytical approach integrating SSM, DQM and numerical inversion method of Laplace transform has been proposed to analyse the transient responses of FGPM cylindrical panels with various boundary conditions. The comparisons of this semi-analytical approach to ANSYS's results in this paper could be more convincing.

\section{Geometry description}

A linear elastic FGPM cylindrical panel is established, and its length $l$, central angle $\alpha$, outer radius $a$ and inner radius $b$ are all considered. The model and its coordinate system are depicted in Fig. 1. The mechanical material properties of the panel vary gradually along the radial direction in an arbitrary manner. By extending the laminated plate model to FGPM cylindrical panel, the panel consists of $K$-layers of graded materials along the radial directions. 


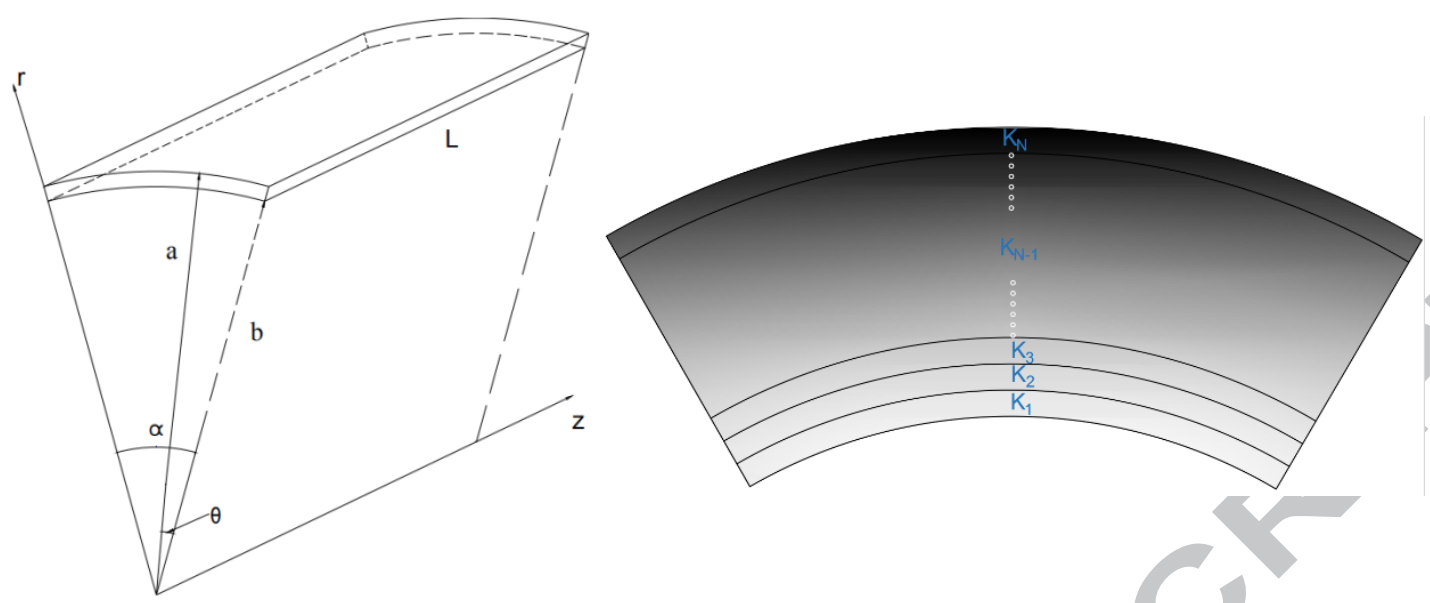

Fig. 1 The FGPM cylindrical panel and its cross section.

Dielectric coefficient and piezoelectric coefficient are important parts of the constitutive equations for stress and electric displacement components. So the linear constitutive relationships can be expressed as

$$
\{\sigma\}=[C]\{\varepsilon\}-[e]^{T}\{E\} \quad\{D\}=[e]\{\varepsilon\}+[\eta]\{E\}
$$

Where $\{\sigma\}$ is the component of stress; $\{\varepsilon\}$ is the component of strain; $\{E\}$ is the electric field; $\{D\}$ is the component of electric displacement. In this cylindrical coordinate system, those components can be given by

$$
\begin{gathered}
\{\sigma\}=\left[\begin{array}{cccccc}
\sigma_{r} & \sigma_{\theta} & \sigma_{z} & \tau_{z \theta} & \tau_{r z} & \tau_{r \theta}
\end{array}\right]^{T} \\
\{E\}=\left[\begin{array}{lllll}
E_{r} & E_{\theta} & E_{z}
\end{array}\right]^{T} \\
\{\varepsilon\}=\left[\begin{array}{llllll}
\varepsilon_{r} & \varepsilon_{\theta} & \varepsilon_{z} & \gamma_{z \theta} & \gamma_{r z} & \gamma_{r \theta}
\end{array}\right]^{T} \\
\{D\}=\left[\begin{array}{llll}
D_{r} & D_{\theta} & D_{z}
\end{array}\right]^{T}
\end{gathered}
$$

Generally, the electric field is related to the electrical potential $\psi$. So it is introduced that

$$
\left[\begin{array}{lll}
E_{r} & E_{\theta} & E_{z}
\end{array}\right]=\left[\begin{array}{lll}
-\frac{\partial \psi}{\partial r} & -\frac{\partial \psi}{r \partial \theta} & -\frac{\partial \psi}{\partial z}
\end{array}\right]
$$


To satisfy the linear strain-displacement relations, the geometric field variables can be expressed by

$$
\begin{gathered}
\varepsilon_{r}=\frac{\partial u_{r}}{\partial r}, \quad \varepsilon_{\theta}=\frac{1}{r}\left(u_{r}+\frac{\partial u_{\theta}}{\partial \theta}\right), \quad \varepsilon_{z}=\frac{\partial u_{z}}{\partial z} \\
\gamma_{z \theta}=\frac{\partial u_{\theta}}{\partial z}+\frac{1}{r} \frac{\partial u z}{\partial \theta}, \quad \gamma_{r z}=\frac{\partial u_{z}}{\partial r}+\frac{\partial u_{r}}{\partial z}, \quad \gamma_{r \theta}=\frac{1}{r} \frac{\partial u_{r}}{\partial \theta}-\frac{u_{\theta}}{r}+\frac{\partial u_{\theta}}{\partial r}
\end{gathered}
$$

In terms of material properties, $[C],[e]$ and $[\eta]$ represent the elastic stiffness coefficient, piezoelectric constant and dielectric constant, respectively. They are given by

$$
\begin{gathered}
{[C]=\left[\begin{array}{cccccc}
C_{11} & C_{12} & C_{13} & 0 & 0 & 0 \\
C_{12} & C_{22} & C_{23} & 0 & 0 & 0 \\
C_{13} & C_{23} & C_{33} & 0 & 0 & 0 \\
0 & 0 & 0 & C_{44} & 0 & 0 \\
0 & 0 & 0 & 0 & C_{55} & 0 \\
0 & 0 & 0 & 0 & 0 & C_{66}
\end{array}\right]} \\
{[e]=\left[\begin{array}{cccccc}
e_{33} & e_{31} & e_{32} & 0 & 0 & 0 \\
0 & 0 & 0 & 0 & 0 & e_{15} \\
0 & 0 & 0 & 0 & e_{24} & 0
\end{array}\right]} \\
{[\eta]=\left[\begin{array}{ccc}
\eta_{11} & 0 & 0 \\
0 & \eta_{22} & 0 \\
0 & 0 & \eta_{33}
\end{array}\right]}
\end{gathered}
$$

By integrating the relationships into state vectors, $\mathrm{Eq}$ (Error! Reference source not found.) will be rewritten as 


$$
\begin{aligned}
& \sigma_{r}=C_{11} \frac{\partial u_{r}}{\partial r}+C_{12}\left(\frac{u_{r}}{r}+\frac{\partial u_{\theta}}{r \partial \theta}\right)+C_{13} \frac{\partial u_{z}}{\partial z}+e_{33} \frac{\partial \psi}{\partial r} \\
& \sigma_{\theta}=C_{12} \frac{\partial u_{r}}{\partial r}+C_{22}\left(\frac{u_{r}}{r}+\frac{\partial u_{\theta}}{r \partial \theta}\right)+C_{23} \frac{\partial u_{z}}{\partial z}+e_{31} \frac{\partial \psi}{\partial r} \\
& \sigma_{z}=C_{13} \frac{\partial u_{r}}{\partial r}+C_{23}\left(\frac{u_{r}}{r}+\frac{\partial u_{\theta}}{r \partial \theta}\right)+C_{33} \frac{\partial u_{z}}{\partial z}+e_{32} \frac{\partial \psi}{\partial r} \\
& \tau_{\theta z}=C_{44} \frac{\partial u_{\theta}}{\partial z}+C_{44} \frac{\partial u_{z}}{r \partial \theta} \\
& \tau_{r z}=C_{55} \frac{\partial u_{z}}{\partial r}+C_{55} \frac{\partial u_{r}}{\partial z}+e_{24} \frac{\partial \psi}{\partial z} \\
& \tau_{r \theta}=C_{66}\left(\frac{\partial u_{r}}{r \partial \theta}-\frac{u_{\theta}}{r}\right)+C_{66} \frac{\partial u_{\theta}}{\partial r}+e_{15} \frac{\partial \psi}{r \partial \theta} \\
& \left.D_{r}=e_{33} \frac{\partial u_{r}}{\partial r}+e_{31}\left(\frac{u_{r}}{r}+\frac{\partial u_{\theta}}{r \partial \theta}\right)-\eta_{11} \frac{\partial \psi}{\partial r}\right) \\
& D_{\theta}=e_{15}\left(\frac{\partial u_{r}}{r \partial \theta}-\frac{u_{\theta}}{r}\right)+e_{15} \frac{\partial u_{\theta}}{\partial r}-\eta_{22} \frac{\partial \psi}{r \partial \theta} \\
& D_{z}=e_{24}\left(\frac{\partial u_{z}}{\partial r}+\frac{\partial u_{r}}{\partial z}\right)-\eta_{33} \frac{\partial \psi}{\partial z}
\end{aligned}
$$

The equations of equilibrium which is based on the small deformation assumption are introduced as below

$$
\begin{aligned}
& \partial_{r} \sigma_{r}+\frac{\sigma_{r}-\sigma_{\theta}}{r}+\partial_{z} \tau_{r z}+\frac{\partial_{\theta} \tau_{r \theta}}{r}-\rho \partial_{t}^{2} u_{r}=0 \\
& \frac{\partial_{\theta} \sigma_{\theta}}{r}+\frac{2 \tau_{r \theta}}{r}+\partial_{r} \tau_{r \theta}+\partial_{z} \tau_{z \theta}-\rho \partial_{t}^{2} u_{\theta}=0 \\
& \partial_{z} \sigma_{z}+\frac{\tau_{r z}}{r}+\partial_{r} \tau_{r z}+\frac{\partial_{\theta} \tau_{z \theta}}{r}-\rho \partial_{t}^{2} u_{z}=0
\end{aligned}
$$

Since PZT-4 and PZT-5H, which are studied in this paper, are dielectric without free electric charge exsiting across its domain, the right side of Maxwell equation becomes zero. As shown below,

$$
\partial_{r} D_{r}+\frac{D_{r}}{r}+\frac{\partial_{\theta} D_{\theta}}{r}+\partial_{z} D_{z}=0
$$

On the one hand, the circumferential edges $(\theta=0, \alpha)$ of the FGPM cylindrical panel are always simply supported. On the other hand, three kinds of boundary condition are set at length edges $(z=0$, 
l). All four sides are electrically grounded to zero potential. The cases can be expressed as below

Simply supported $(z=0)$-Simply supported $(z=l)$

$$
\begin{aligned}
& \text { at } \quad z=0, \sigma_{z}=u_{r}=u_{\theta}=\psi=0, \\
& \text { at } \quad z=l, \sigma_{z}=u_{r}=u_{\theta}=\psi=0 .
\end{aligned}
$$

Clamped $(z=0)$-Clamped $(z=l)$

$$
\begin{aligned}
& \text { at } \quad z=0, u_{r}=u_{\theta}=u_{z}=\psi=0, \\
& \text { at } \quad z=l, u_{r}=u_{\theta}=u_{z}=\psi=0 .
\end{aligned}
$$

Clamped ( $z=0)$-Simply supported $(z=l)$

$$
\begin{array}{ll}
\text { at } & z=0, u_{r}=u_{\theta}=u_{z}=\psi=0, \\
\text { at } & z=l, \sigma_{z}=u_{r}=u_{\theta}=\psi=0 .
\end{array}
$$

When external loads and initial voltages are applied to the structure, they can be treated as force boundary conditions and voltage boundary conditions.

$$
\begin{aligned}
& \text { at inner surface }(r=b), \sigma_{r}=f_{r b}, \tau_{r \theta}=f_{\theta b}, \tau_{r z}=f_{z b} \text {, and } \psi=\psi_{r b} \\
& \text { at outer surface }(r=a), \sigma_{r}=f_{r a}, \tau_{r \theta}=f_{\theta a}, \tau_{r z}=f_{z a} \text {, and } \psi=\psi_{r a}
\end{aligned}
$$

\section{Discretization of governing equations}

\subsection{Differential quadrature method}

The essence of DQM is to approximate the derivative value of the function at a given node with the weighted sum of the function values at all nodes in the domain. Usually, the choice of weight coefficient and the arrangement of nodes have a great influence on the accuracy of solving the problem. According to the interpolation principle, the weight coefficient in DQM can be directly given by the derivative value of Lagrange interpolation polynomial at nodes [29]. Now, assuming $\tilde{\bar{f}}(\vec{r}, \vec{z}, \vec{s})$ is a continuous function, the $i$-th order partial derivative along the $z$ direction can be expressed by a sum of values in the spatial domain [30-32].

$$
\frac{\partial^{i} \tilde{\bar{f}}\left(\vec{r}, \vec{z}_{m}, \vec{s}\right)}{\partial \vec{z}^{i}}=\sum_{n=1}^{M} A_{m n}^{(i)} \tilde{\bar{f}}\left(\vec{r}, \vec{z}_{n}, \vec{s}\right)
$$


where

$$
\begin{aligned}
A_{m n}^{(1)} & =\frac{\prod_{k=1, k \neq m}^{M}\left(x_{m}-x_{k}\right)}{\left(x_{m}-x_{n}\right) \prod_{k=1, k \neq n}^{M}\left(x_{n}-x_{k}\right)}, \\
A_{m n}^{(i)} & =i\left[A_{m m}^{(i-1)} A_{m n}^{(1)}-\frac{A_{m n}^{(i-1)}}{x_{m}-x_{n}}\right], i=2,3, \ldots, M-1, \text { and } m \neq n \\
A_{m m}^{(i)} & =-\sum_{n=1, n \neq m}^{M} A_{m n}^{(i)}, m=1,2, \ldots, M, i=2,3, \ldots, M-1
\end{aligned}
$$

where $m$ is from 1 to sampling points $M ; n$ is from 1 to $M-1 ; A_{m n}^{(i)}$ is the weighting coefficients $[33,34]$.

\subsection{Laplace transform and its numerical inversion method}

The Laplace transform, which is very effective in solving complex differential equations, is widely carried out to deal with the problems of structural dynamics $[35,36]$. The Laplace transform is then defined by:

$$
\tilde{f}(s)=\mathcal{L}[f(t)]=\int_{0}^{\infty} f(t) \exp (-s t) \mathrm{d} t,
$$

where $\mathcal{L}$ and the over-script - represent the Laplace transform and transformed function, respectively; $s$ is the replacement variable of $t$ in the Laplace domain.

To seek a solution for the complicated dynamic problems, Durbin's [37] numerical inversion method was proposed which always generates almost the same results as that given by analytical inversion methods in a short time range [38]. The Laplace numerical inversion approach can be expressed as following formula.

$$
f(\vec{t})=\frac{2 \exp (\alpha \vec{t})}{T}\left\{\frac{\tilde{f}(\alpha)}{2}+\sum_{k=1}^{K} \operatorname{Re}[\tilde{f}(\alpha+k \pi \mathrm{i} / T)] \cos (k \pi \vec{t} / T)\right\}
$$

where $\alpha=5 / T, T=5 \times T_{d}, K$ is a sufficient number, and $T_{d}$ is the observation period.

To separate the variable $\theta$, Fourier series are employed to expand the components of stress and displacement into the trigonometric formulations. The wave number is $j$, and the panel's sector angle is $\alpha$ [38]. 


$$
\left\{\begin{array}{l}
\vec{\sigma}_{r}(\vec{r}, \theta, \vec{z}, t) \\
\vec{u}_{r}(\vec{r}, \theta, \vec{z}, t) \\
\vec{u}_{\theta}(\vec{r}, \theta, \vec{z}, t) \\
\vec{u}_{z}(\vec{r}, \theta, \vec{z}, t) \\
\vec{\tau}_{r z}(\vec{r}, \theta, \vec{z}, t) \\
\vec{\tau}_{r \theta}(\vec{r}, \theta, \vec{z}, t) \\
\vec{\sigma}_{\theta}(\vec{r}, \theta, \vec{z}, t) \\
\vec{\sigma}_{z}(\vec{r}, \theta, \vec{z}, t) \\
\vec{\tau}_{z \theta}(\vec{r}, \theta, \vec{z}, t) \\
\vec{D}_{r}(\vec{r}, \theta, \vec{z}, t) \\
\vec{D}_{\theta}(\vec{r}, \theta, \vec{z}, t) \\
\vec{D}_{z}(\vec{r}, \theta, \vec{z}, t)
\end{array}\right\}=\sum_{j=0}^{\infty}\left\{\begin{array}{c}
\bar{\sigma}_{r}(\vec{r}, \vec{z}, t) \sin \left(L^{*} \theta\right) \\
\bar{u}_{r}(\vec{r}, \vec{z}, t) \sin \left(L^{*} \theta\right) \\
\bar{u}_{\theta}(\vec{r}, \vec{z}, t) \cos \left(L^{*} \theta\right) \\
\bar{u}_{z}(\vec{r}, \vec{z}, t) \sin \left(L^{*} \theta\right) \\
\bar{\tau}_{r z}(\vec{r}, \vec{z}, t) \sin \left(L^{*} \theta\right) \\
\bar{\tau}_{r \theta}(\vec{r}, \vec{z}, t) \cos \left(L^{*} \theta\right) \\
\bar{\sigma}_{\theta}(\vec{r}, \vec{z}, t) \sin \left(L^{*} \theta\right) \\
\bar{\sigma}_{z}(\vec{r}, \vec{z}, t) \sin \left(L^{*} \theta\right) \\
\bar{\tau}_{z \theta}(\vec{r}, \vec{z}, t) \cos \left(L^{*} \theta\right) \\
\bar{D}_{r}(\vec{r}, \vec{z}, t) \sin \left(L^{*} \theta\right) \\
\bar{D}_{\theta}(\vec{r}, \vec{z}, t) \cos \left(L^{*} \theta\right) \\
\bar{D}_{z}(\vec{r}, \vec{z}, t) \sin \left(L^{*} \theta\right)
\end{array}\right\},
$$

where $L^{*}=j \pi / \alpha$

By substituting Eqs. (Error! Reference source not found.,Error! Reference source not

found.), the governing equations can be obtained in the framework of SSM. After employing the

Fourier expansion and Laplace transform, the fundamental equations are derived as

$$
\begin{aligned}
& \frac{d \tilde{\bar{\sigma}}_{r}}{d r}=a\left(\rho s^{2}+\frac{C_{33} \delta_{10}-C_{13} \delta_{3}+e_{33} \delta_{9}}{r^{2}}\right) \tilde{\bar{u}}_{r}-\frac{a j \pi\left(-C_{13} \delta_{3}+e_{33} \delta_{9}+C_{33} \delta_{10}\right)}{r^{2} \alpha} \tilde{\bar{u}}_{\theta} \\
& +\frac{a\left(-C_{23} \delta_{3}-e_{33} \delta_{7}+C_{33} \delta_{8}\right)}{l r} \frac{\partial \tilde{\bar{u}}_{z}}{\partial z}+\frac{a\left(\delta_{1}+\delta_{2}\right)}{r} \tilde{\bar{\sigma}}_{r}-\frac{a}{l} \frac{\partial \tilde{\bar{\tau}}_{r z}}{\partial z}+\frac{a j \pi}{r \alpha} \tilde{\bar{\tau}}_{r \theta}-\frac{a \delta_{4}}{r} \tilde{\bar{D}}_{r} \\
& \frac{d \tilde{\bar{u}}_{r}}{d r}=-\frac{a \delta_{3}}{r} \tilde{\bar{u}}_{r}+\frac{a j \pi \delta_{3}}{r \alpha} \tilde{\bar{u}}_{\theta}-\frac{a \delta_{5}}{l} \frac{\partial \tilde{\bar{u}}_{z}}{\partial z}+\frac{a \eta_{33}}{e_{33}^{2}+C_{33} \eta_{33}} \tilde{\bar{\sigma}}_{r}+\frac{a e_{33}}{e_{33}^{2}+C_{33} \eta_{33}} \tilde{\bar{D}}_{r} \\
& \frac{d \tilde{\bar{u}}_{\theta}}{d r}=-\frac{a j \pi}{r \alpha} \tilde{\bar{u}}_{r}+\frac{a}{r} \tilde{\bar{u}}_{\theta}+\frac{a}{C_{55}} \tilde{\overline{\bar{\tau}}}_{r \theta}-\frac{a j \pi e_{15}}{r \alpha C_{55}} \tilde{\bar{\psi}}_{r} \\
& \frac{d \tilde{\bar{u}}_{z}}{d r}=-\frac{a}{l} \frac{\partial \tilde{\bar{u}}_{r}}{\partial z}+\frac{a}{C_{55}} \tilde{\bar{\tau}}_{r z}-\frac{a e_{24}}{l C_{44}} \frac{\partial \tilde{\bar{\psi}}_{r}}{\partial z} \\
& \frac{d \tilde{\bar{\tau}}_{r z}}{d r}=\frac{a\left(C_{23} \delta_{3}-C_{33} \delta_{8}+e_{33} \delta_{7}\right)}{l r} \frac{\partial \tilde{\bar{u}}_{r}}{\partial z}+\frac{a j \pi\left(C_{12}+C_{66}-C_{23} \delta_{3}+e_{32} \delta_{4}\right)}{\operatorname{lr} \alpha} \frac{\partial \tilde{\bar{u}}_{\theta}}{\partial z} \\
& +a\left(\rho s^{2}+\frac{j^{2} \pi^{2} C_{66}}{r^{2} \alpha^{2}}\right) \tilde{\bar{u}}_{z}+\frac{a\left(-C_{33}+e_{32} \delta_{6}+C_{23} \delta_{5}\right)}{l^{2}} \frac{\partial \tilde{\bar{u}}_{z}}{\partial z}-\frac{a \delta_{5}}{l} \frac{\partial \tilde{\bar{\sigma}}_{r}}{\partial z}-\frac{a}{r} \tilde{\bar{\tau}}_{r z}+\frac{a \delta_{6}}{l} \frac{\partial \tilde{\bar{D}}_{r}}{\partial z}
\end{aligned}
$$




$$
\begin{aligned}
\frac{d \tilde{\bar{\tau}}_{r \theta}}{d r}=- & \frac{a j \pi\left(-C_{13} \delta_{3}+C_{33} \delta_{10}+e_{33} \delta_{9}\right)}{r^{2} \alpha} \tilde{\bar{u}}_{r}+a\left(\rho s^{2}+\frac{j^{2} \pi^{2}\left(-C_{13} \delta_{3}+e_{33} \delta_{9}+C_{33} \delta_{10}\right)}{r^{2} \alpha^{2}}\right) \tilde{\bar{u}}_{\theta} \\
& -\frac{a C_{66}}{l^{2}} \frac{\partial \tilde{\bar{u}}_{\theta}}{\partial z}-\frac{a j \pi\left(C_{12}+C_{66}-C_{23} \delta_{3}+e_{32} \delta_{4}\right)}{l r \alpha} \frac{\partial \tilde{\bar{u}}_{z}}{\partial z}-\frac{a j \pi \delta_{3}}{r \alpha} \tilde{\bar{\sigma}}_{r}-\frac{2 a}{r} \tilde{\bar{\tau}}_{r \theta}+\frac{a j \pi \delta_{4}}{r \alpha} \tilde{\bar{D}}_{r} \\
\frac{d \tilde{\bar{D}}_{r}}{d r}=- & \frac{a e_{24}}{l C_{44}} \frac{\partial \tilde{\bar{\tau}}_{r z}}{\partial z}+\frac{a j \pi e_{15}}{r \alpha C_{55}} \tilde{\bar{\tau}}_{r \theta}-\frac{a}{r} \tilde{\bar{D}}_{r}-\frac{a j^{2} \pi^{2} \delta_{11}\left(e_{33}^{2}+C_{33} \eta_{33}\right)}{r^{2} \alpha^{2} C_{55}} \tilde{\overline{\bar{\psi}}}_{r}+\frac{a \delta_{12}\left(e_{33}^{2}+C_{33} \eta_{33}\right)}{l^{2} C_{44}} \frac{\partial \tilde{\bar{\psi}}_{r}}{\partial z} \\
& \frac{d \tilde{\bar{\psi}}_{r}}{d r}=\frac{a \delta_{4}}{r} \tilde{\bar{u}}_{r}-\frac{a j \pi \delta_{4}}{r \alpha} \tilde{\bar{u}}_{\theta}+\frac{a \delta_{6}}{r} \frac{\partial \tilde{\bar{u}}_{z}}{\partial z}+\frac{a e_{33}}{e_{33}^{2}+C_{33} \eta_{33}} \tilde{\overline{\bar{\sigma}}}_{r}-\frac{a C_{33}}{e_{33}^{2}+C_{33} \eta_{33}} \tilde{\overline{\bar{D}}}_{r}
\end{aligned}
$$

where

$$
\begin{aligned}
& \frac{e_{31} e_{33}-e_{33}^{2}}{e_{33}^{2}+C_{33} \eta_{33}}=\delta_{1} \frac{\left(C_{13}-C_{33}\right) \eta_{33}}{e_{33}^{2}+C_{33} \eta_{33}}=\delta_{2} \frac{e_{31} e_{33}+C_{13} \eta_{33}}{e_{33}^{2}+C_{33} \eta_{33}}=\delta_{3} \frac{C_{33} e_{31}-C_{13} e_{33}}{e_{33}^{2}+C_{33} \eta_{33}}=\delta_{4} \\
& \frac{e_{32} e_{33}+C_{23} \eta_{33}}{e_{33}^{2}+C_{33} \eta_{33}}=\delta_{5} \frac{C_{33} e_{32}-C_{23} e_{33}}{e_{33}^{2}+C_{33} \eta_{33}}=\delta_{6} \frac{C_{13} e_{32}-C_{12} e_{33}}{e_{33}^{2}+C_{33} \eta_{33}}=\delta_{7} \frac{e_{31} e_{32}+C_{12} \eta_{33}}{e_{33}^{2}+C_{33} \eta_{33}}=\delta_{8} \\
& \frac{C_{22} e_{33}-C_{13} e_{31}}{e_{33}^{2}+C_{33} \eta_{33}}=\delta_{9} \quad \frac{e_{31}^{2}+C_{22} \eta_{33}}{e_{33}^{2}+C_{33} \eta_{33}}=\delta_{10} \quad \frac{e_{15}^{2}+C_{55} \eta_{11}}{e_{33}^{2}+C_{33} \eta_{33}}=\delta_{11} \quad \frac{e_{24}^{2}+C_{44} \eta_{22}}{e_{33}^{2}+C_{33} \eta_{33}}=\delta_{12}
\end{aligned}
$$

The other five variables are induced variables which are determined by the state space vectors.

So the five variables can be expressed as follows

$$
\begin{aligned}
\widetilde{\bar{\sigma}}_{z}= & \frac{\left(-C_{23} \delta_{3}-e_{33} \delta_{7}+C_{33} \delta_{8}\right) \tilde{\bar{u}}_{r}}{r}+\frac{j \pi\left(C_{23} \delta_{3}-C_{33} \delta_{8}+e_{33} \delta_{7}\right) \tilde{\bar{u}}_{\theta}}{r \alpha}+\frac{\left(C_{33}-C_{23} \delta_{5}-e_{32} \delta_{6}\right)}{l} \frac{\partial \tilde{\bar{u}}_{z}}{\partial z} \\
& +\delta_{5} \widetilde{\bar{\sigma}}_{r}-\delta_{6} \widetilde{\bar{D}}_{r} \\
\widetilde{\bar{\sigma}}_{\theta}= & \frac{\left(C_{33}-C_{13} \delta_{3}+e_{33} \delta_{9}\right) \tilde{\bar{u}}_{r}}{r}-\frac{j \pi\left(C_{33}-C_{13} \delta_{3}+e_{33} \delta_{9}\right) \tilde{\bar{u}}_{\theta}}{r \alpha}+\frac{\left(-C_{23} \delta_{3}-e_{33} \delta_{7}+C_{33} \delta_{8}\right)}{l} \frac{\partial \tilde{\bar{u}}_{z}}{\partial z} \\
& +\delta_{3} \widetilde{\bar{\sigma}}_{r}-\delta_{4} \widetilde{\bar{D}}_{r} \\
\tilde{\bar{\tau}}_{z \theta}= & \frac{C_{66}}{l} \frac{\partial \tilde{\bar{u}}_{\theta}}{\partial z}+\frac{j \pi C_{66} \tilde{\bar{u}}_{z}}{r \alpha} \\
\widetilde{\bar{D}}_{\theta}= & \frac{e_{15} \tilde{\bar{\tau}}_{r \theta}}{C_{55}}-\frac{j \pi \delta_{11}\left(e_{33}^{2}+C_{33} \eta_{33}\right) \widetilde{\bar{\psi}}_{r}}{r \alpha C_{55}} \\
\widetilde{\bar{D}}_{z}= & -\frac{\left(e_{24}^{2}+C_{44} \eta_{22}\right)}{C_{44} l} \frac{\partial \widetilde{\bar{\psi}}_{r}}{\partial z}+\frac{e_{24} \tilde{\bar{\tau}}_{r z}}{C_{44}}
\end{aligned}
$$


As $M$ is the sum of sample points, $m^{\text {th }}$ is the location of calculating the sample point. By applying DQM on the equation Error! Reference source not found.Eq.(Error! Reference source not found.,Error! Reference source not found.), the new state space equations can be rewritten in the Appendix.

A set of dimensionless coordinates and elastic field variables are defined as

$$
\begin{aligned}
& \vec{C}_{i j}=C_{i j} / C_{33}, \vec{r}=r / a, \vec{z}=z / l, \vec{t}=c t / a, \vec{s}=s a / c, \vec{\psi}=\psi \cdot \eta_{33},\left\{\vec{u}_{r}, \vec{u}_{\theta}, \vec{u}_{z}\right\}=\left\{u_{r}, u_{\theta}, u_{z}\right\} / a, \\
& \left\{\vec{\sigma}_{r}, \vec{\sigma}_{\theta}, \vec{\sigma}_{z}, \vec{\tau}_{r \theta}, \vec{\tau}_{z \theta}, \vec{\tau}_{r z}\right\}=\left\{\sigma_{r}, \sigma_{\theta}, \sigma_{z}, \tau_{r \theta}, \tau_{z \theta}, \tau_{r z}\right\} / C_{33}, \quad\left\{\vec{D}_{r}, \vec{D}_{\theta}, \vec{D}_{z}\right\}=\left\{D_{r}, D_{\theta}, D_{z}\right\} / e_{33}
\end{aligned}
$$

where $i, j=1,2, \ldots, 6$ and $c$ is the wave velocity.

$$
c=\left(C_{33} / \rho\right)^{1 / 2}
$$

\section{Equation simplification}

As shown in Eq.(Error! Reference source not found.), the differential equations with so many variables are too complex to be solved by analytical approaches. Hence, for the sake of simplification, an approximate method is performed. Eq.(Error! Reference source not found.) is supposed to be rewritten by the form of a matrix

$$
\frac{\mathrm{d} \tilde{\bar{Q}}(\lambda)}{\mathrm{d} \lambda}=\mathbf{H} \cdot \tilde{\overline{\mathrm{Q}}}(\lambda)
$$

where $\lambda$ refers to the distance between the origin of coordinate and observing point; $\tilde{\overline{\mathbf{Q}}}=\left\{\tilde{\overline{\boldsymbol{\sigma}}}_{r}, \tilde{\overline{\mathbf{u}}}_{r}, \tilde{\overline{\mathbf{u}}}_{\theta}, \tilde{\overline{\mathbf{u}}}_{z}, \tilde{\overline{\boldsymbol{\tau}}}_{r z}, \tilde{\overline{\boldsymbol{\tau}}}_{r \theta}, \tilde{\bar{D}}_{r}, \tilde{\bar{\psi}}_{r}\right\}, \quad \tilde{\overline{\mathbf{\sigma}}}_{r}=\left\{\tilde{\bar{\sigma}}_{r 1}, \ldots \tilde{\overline{\bar{\sigma}}}_{r M}\right\}^{\mathrm{T}}, \quad \tilde{\overline{\mathbf{u}}}_{r}=\left\{\tilde{\bar{u}}_{r 1}, \ldots \tilde{\bar{u}}_{r M}\right\}^{\mathrm{T}}, \ldots$ The constitution of matrix $H$ can be found in Error! Reference source not found.. After applying different boundary conditions to the Eqs. (Error! Reference source not found.)-(Error! Reference source not found.), the various $H$ are given in Error! Reference source not found..

The expression of Eq. (Error! Reference source not found.) can be given by

$$
\tilde{\overline{\mathrm{Q}}}(\lambda)=\exp \left[\mathbf{H}_{k}\left(\lambda-\lambda_{k-1}\right)\right] \cdot \tilde{\mathrm{Q}}\left(\lambda_{k-1}\right) \cdot\left(0 \leq \lambda \leq \frac{h}{b}\right)
$$


where $h=a$ - $b$, Eq. (Error! Reference source not found.) at $\lambda=\lambda_{k}$ yields

$$
\tilde{\overline{\mathrm{Q}}}\left(\lambda_{k}\right)=\exp \left(\mathbf{H} h_{k}\right) \cdot \tilde{\overline{\mathrm{Q}}}\left(\lambda_{k-1}\right)
$$

where $h_{k}$ is the thickness of $k^{\text {th }}$ layer.

Subsequently,

$$
\tilde{\overline{\mathrm{Q}}}\left(\lambda_{k+1}\right)=\exp \left(\mathbf{H}_{k+1} h_{k+1}\right) \cdot \tilde{\overline{\mathrm{Q}}}\left(\lambda_{k}\right)=\exp \left(\mathbf{H}_{k+1} h_{k+1}\right) \cdot \exp \left(\mathbf{H}_{k} h_{k}\right) \cdot \tilde{\overline{\mathrm{Q}}}\left(\lambda_{k-1}\right)
$$

For all $K$ layers have the same behaviour, the FGPM cylindrical panel's state vectors at the outer surface can be determined by the inner surface

$$
\tilde{\overline{\mathrm{Q}}}(a)=\mathbf{T}(a-b) \cdot \tilde{\overline{\mathrm{Q}}}(b)
$$

where

$$
\begin{aligned}
& \mathbf{T}(h)=\prod_{k=K}^{1} \exp \left(\mathbf{H}_{k} h_{k}\right) \\
& \mathbf{T}(h)=T_{8 \times 8} \quad(m, n=1,2, \ldots, 8)
\end{aligned}
$$

\section{Solutions and discussions}

Firstly, compared with data obtained by other theoretical methods, the natural frequencies of an FGPM cylindrical panel are investigated for different FG index $\gamma$. Subsequently, the displacements and electric potential along $r$ - direction obtained by the semi-analytical method are compared with FEM to validate the method. And then, the research regarding convergence is carried out for different cases. Finally, this allows the effects of cylindrical panel's initial electric potential, FG index and central angle to be studied.

\subsection{Nature frequencies study}

In comparison with previous results in Reference [39], the natural frequencies of the FGPM cylindrical panel are investigated. The geometric sizes of the cylindrical panel are various in Table. 2 with simply supported boundary condition and different index $\gamma$. The compositions of this FGPM panel are PZT-4 and PZT-5H, respectively. The constituents of the two kinds of material are described in Table. 1 [40]. 
Table. 1 Fundamental material properties along the radial direction.

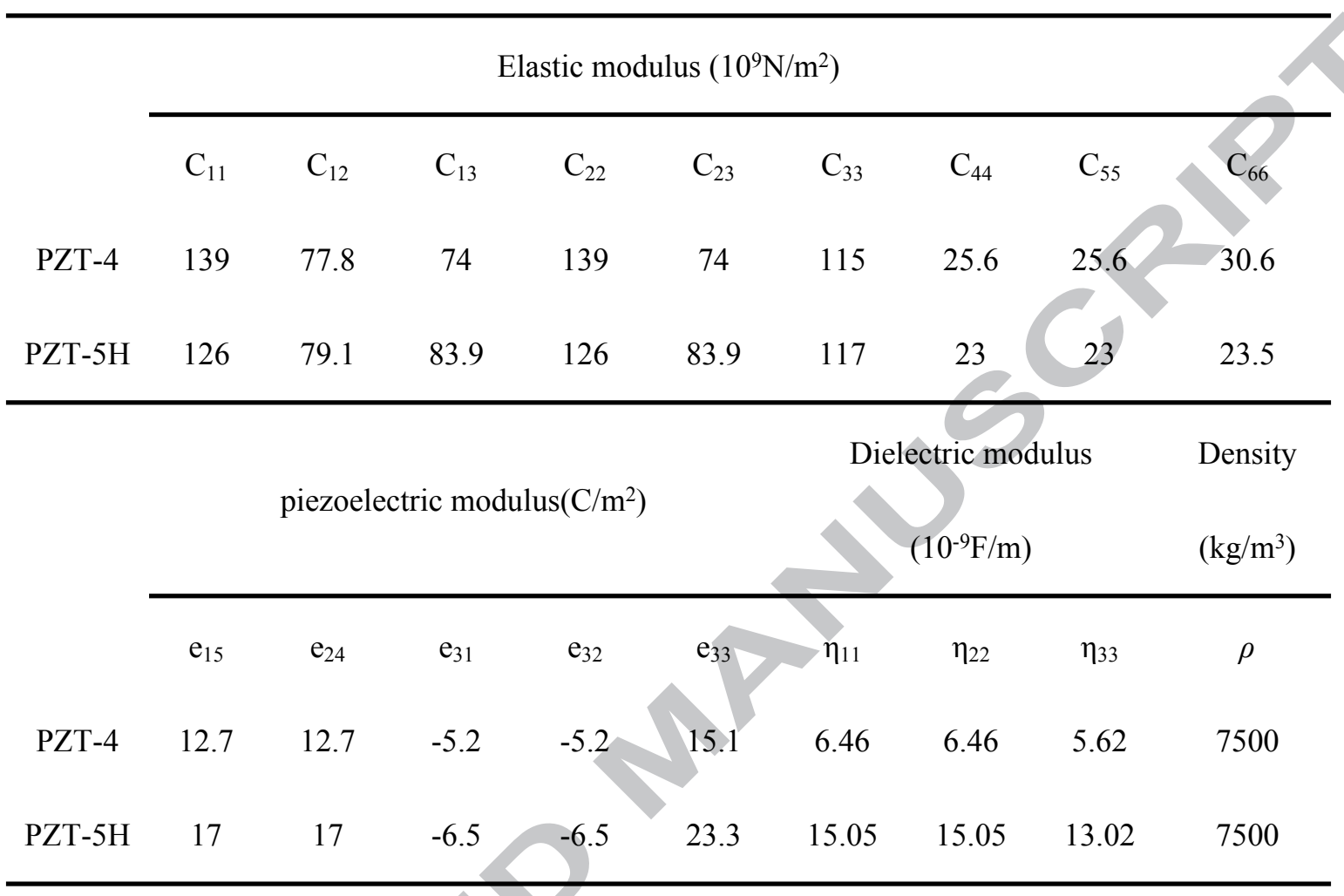

The purpose of free vibration analysis is to verify the reliability of the proposed approach. The results of the first mode frequencies are shown in Table. 2 for different FG index under different geometric sizes along with those of M.Bodaghi [39]. It is indicated that the values obtained by this approach agree well with M.Bodaghi's [39] method. In the Table. 2, non-dimensional frequency parameter is given by $\omega^{*}=\omega(a-b) \sqrt{\rho_{5 H} / C_{11,5 H}}$ in which " $5 \mathrm{H}$ " means the property of PZT-5H.

Table. 2 Natural frequencies of the first mode for FGPM cylindrical panels.

\begin{tabular}{|c|c|c|c|c|c|c|c|}
\hline \multirow{2}{*}{$(a-b) / R$} & \multirow{2}{*}{$\mathrm{L} / \mathrm{R}$} & \multicolumn{6}{|c|}{$\gamma$} \\
\hline & & & 0 & 0.5 & 1.0 & 3.0 & 5.0 \\
\hline \multirow{3}{*}{0.05} & & M.Bodaghi & 0.04597 & 0.04350 & 0.04280 & 0.04185 & 0.04152 \\
\hline & 0.5 & & & & & & \\
\hline & & Present & 0.04612 & 0.04318 & 0.04263 & 0.04190 & 0.04178 \\
\hline
\end{tabular}




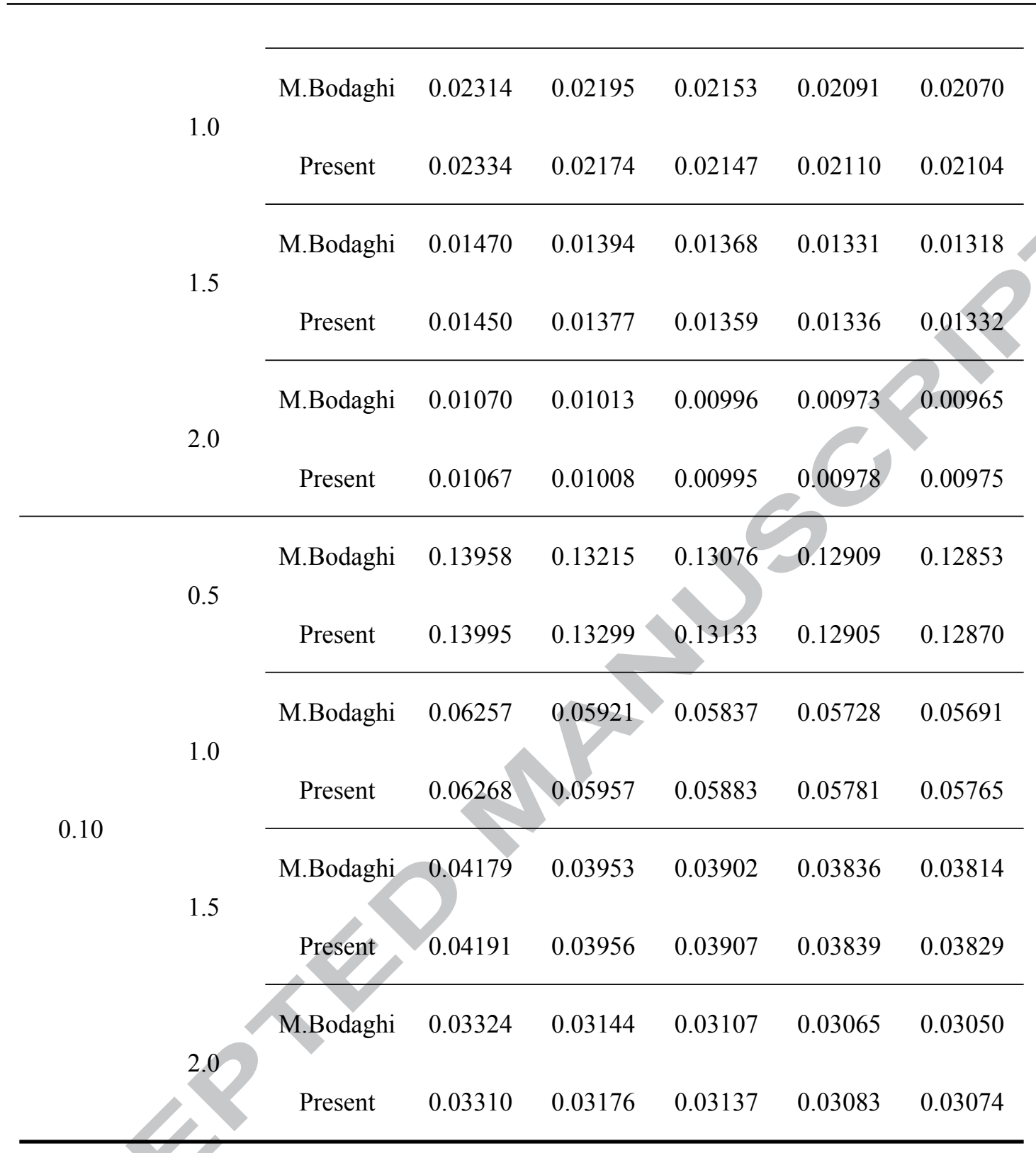

In the table $R=(a+b) / 2$. It can be concluded that the fundamental frequency increases with the increase of thickness and the decrease of length, respectively. Also, it can be found that the natural frequency decreases by increasing the material power law index due to changes in the material properties from PZT-4 to PZT-5H.

\subsection{Dynamic response analysis}

In order to further demonstrate the validation of the proposed approach, its displacements and electric potential along the radial direction are compared with the results by commercial software ANSYS. The cases with various variables are given in Table. 3 . 
Table. 3 The cases with different variables and boundaries.

\begin{tabular}{ccccccc}
\hline $\begin{array}{c}\text { Case } \\
\text { number }\end{array}$ & $\begin{array}{c}\text { Variation law of } \\
\text { material } \\
\text { properties }\end{array}$ & $\begin{array}{c}\text { central } \\
\text { angle } \alpha\end{array}$ & $\begin{array}{c}\text { FG } \\
\text { index } \gamma\end{array}$ & $\begin{array}{c}\text { Outer } \\
\text { Radius } \\
a(\mathrm{~m})\end{array}$ & $\begin{array}{c}\text { inner } \\
\text { Radius } \\
b(\mathrm{~m})\end{array}$ & $\begin{array}{c}\text { Boundary } \\
\text { conditions } \\
\text { at } z=0, l\end{array}$ \\
\hline 1 & Exponential & $30^{\circ}$ & 2 & 1 & 0.9 & $\mathrm{C}-\mathrm{C}$ \\
2 & Power & $60^{\circ}$ & 0.2 & 1 & 0.8 & S-S \\
3 & Power & $90^{\circ}$ & 0.5 & 1 & 0.8 & $\mathrm{C}-\mathrm{S}$ \\
\hline
\end{tabular}

For the orthotropic properties, the stiffness coefficients, mass density, piezoelectric constants and dielectric constants are supposed to obey the identical exponential law with the thickness coordinates. The material properties along the radial direction are given by the following equation [41]

$$
\left\{C_{i j}, \rho, e_{m n}, \eta_{r s}\right\}=\left\{\hat{C}_{i j}, \hat{\rho}, \hat{e}_{m n}, \hat{\eta}_{r s}\right\} \times \exp [\gamma(a-r) /(a-b)]
$$

where the subscript $i$ and $j$ range from 1 to $6 ; n$ ranges from 1 to $5 ; m, r$ and $s$ range from 1 to 3. In our studies, the material properties of this FGPM cylindrical panel are made up of PZT-4 and PZT-5H. Moreover, the distribution of volume fractions along the radial direction which obeys the power laws can be defined as

$$
\begin{aligned}
& V_{P}(r)=[(a-r) /(a-b)]^{\gamma}, \\
& V_{5 H}(r)=1-[(a-r) /(a-b)]^{\gamma}
\end{aligned}
$$

P means PZT-4 and 5H represents PZT-5H. Therefore, the material properties of this FGPM cylindrical panel are derived as

$$
\left\{C_{i j}, \rho, e_{m n}, \eta_{r s}\right\}=\left\{C_{i j, P}, \rho_{P}, e_{m n, P}, \eta_{r s, P}\right\} V_{P}(r)+\left\{C_{i j, 5 H}, \rho_{5 H}, e_{m n, 5 H}, \eta_{r s, 5 H}\right\} V_{5 H}(r)
$$

Then for each layer, the distribution properties can be defined as:

$$
\left\{C_{i j}, \rho, e_{m n}, \eta_{r s}\right\}_{k}=\frac{\int_{z_{k-1}}^{z_{k}}\left\{C_{i j}, \rho, e_{m n}, \eta_{r s}\right\}(r) \mathrm{d} r}{r_{k}-r_{k-1}}
$$

where $k=1,2, \ldots, K$. 
In order to compare with the results of ANSYS, the applying load is given as below [42]

$$
\text { at } r=a, \sigma_{r}=S_{0} C_{33, P} \sin \left(\frac{j \pi}{\alpha} \theta\right) \exp \left(-t c_{P} / l\right)
$$

The initial electric potential in inter surface and outer surface are equal to zero.

To show the effects of initial electric potential, the cylindrical panel in case 2 is subjected to the following electromechanical load

at $r=a$

$$
\begin{aligned}
& \sigma_{r}=S_{0} C_{33, P} \sin \left(\frac{j \pi}{\alpha} \theta\right) \exp \left(-t c_{P} / l\right), \\
& \psi_{r}=V_{0, a} \sin \left(\frac{j \pi}{\alpha} \theta\right) / \eta_{33, P},
\end{aligned}
$$

at $r=b$

$$
\begin{aligned}
& \sigma_{r}=0, \\
& \psi_{r}=V_{0, b} \sin \left(\frac{j \pi}{\alpha} \theta\right) / \eta_{33, P}
\end{aligned}
$$

At first, the layer number $K$ and sampling point $M$ are 4 and 21, respectively. $S_{0}$ is given to 0.001. Solid 64 of the structure elements are used in the finite element analysis. The central position $(r=(a+b) / 2, \theta=0, z=l / 2)$ is chosen to calculate the results. The behaviour of FGPM cylindrical panel under mechanical load without initial voltage is presented. Along the radial direction, the numerical results of displacement and electric potential between the proposed approach and FEM are compared with each other in Fig. 2. It is shown that the results predicted by the two methods have a good agreement. Moreover, the numerical solutions are independent of conditions determined by geometry, FG index, and other boundary conditions.
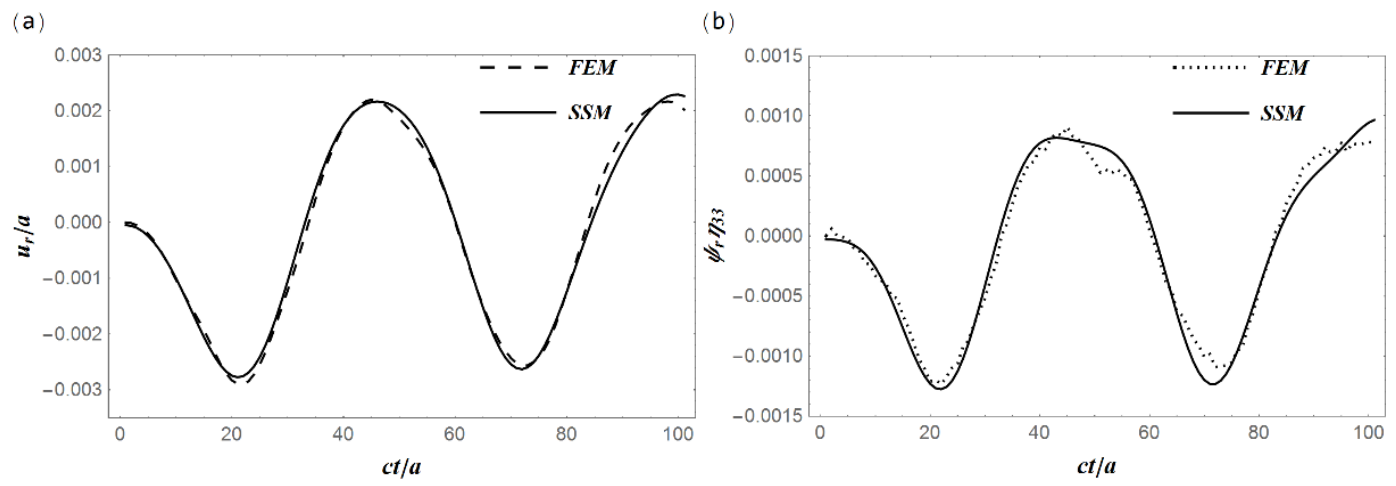


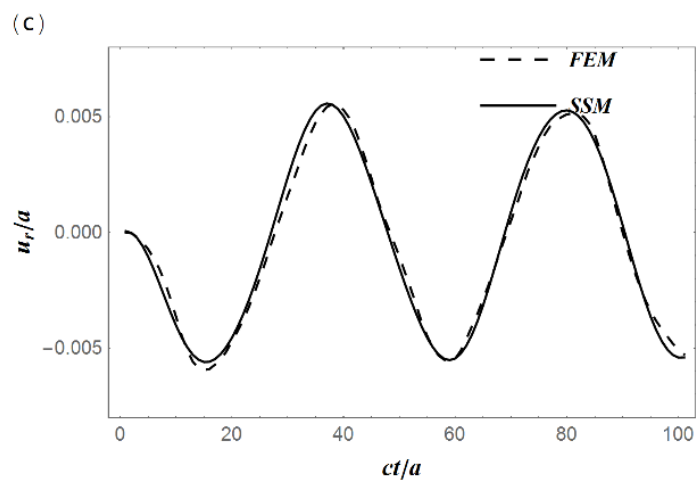

(d)
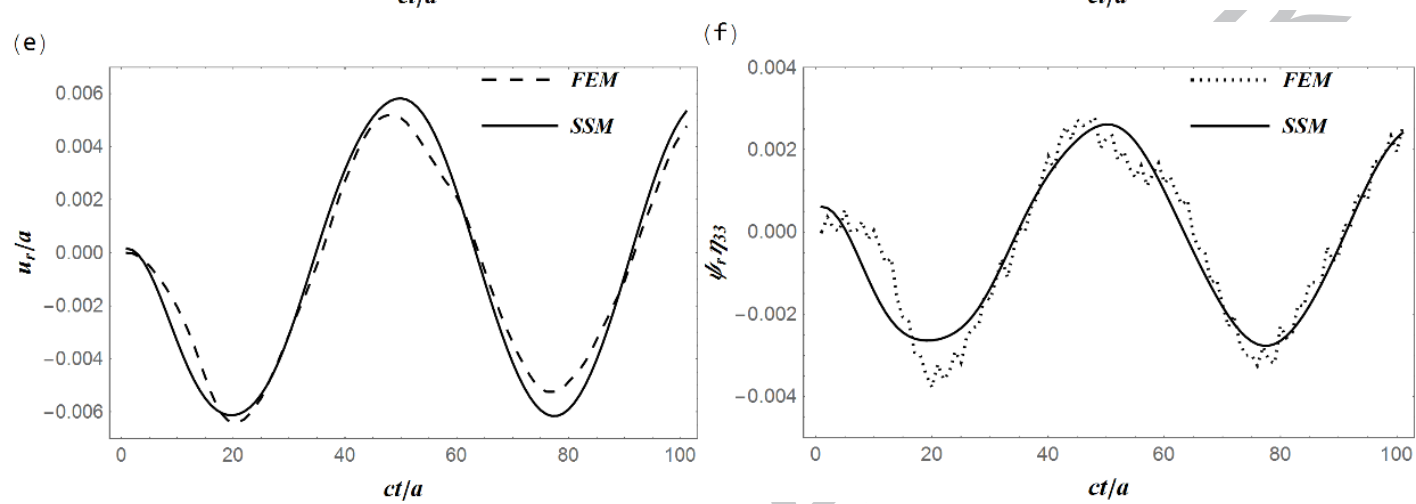

Fig. 2 Deflection and electric potential (a,b) case 1; (c,d) case 2;(e,f) case 3.

Obviously, a strong correlation between deformation and electric potential can be observed.

This correlation is shown in Fig. 2 with three different types of boundary conditions.

\section{3 convergence analysis}

When it comes to convergence study, the analysis for different numbers of sampling points $M$ along the length direction and layer numbers $K$ along the radial direction are carried out. The values of $M$ are 5,9, 13 and 21, respectively. And a series of layer numbers are employed in order to analyse the convergence rate with simply supported in case 2 .

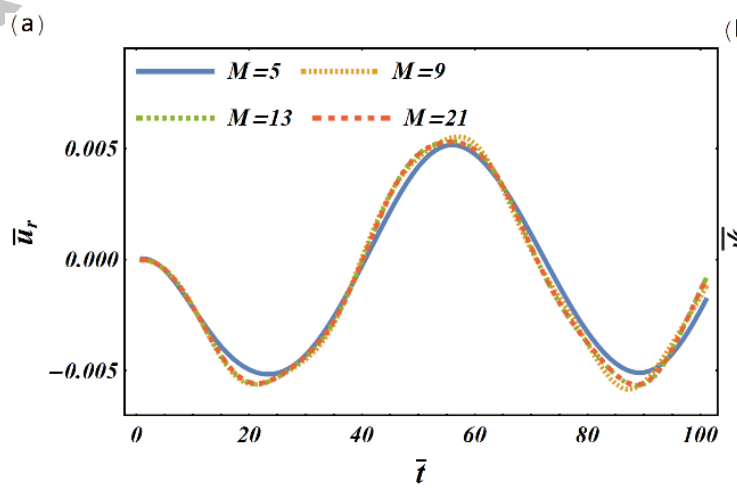

(b)

Fig. 3 Deflection and electric potential with sampling points. 
The relations between the normalised deflection and electric potential with time history at the central position are shown in Fig. 3. It is indicated that the more sampling point numbers, the more accurate the results will be. When the numbers of sampling points are at a relatively large level, the results do not change much. It also displays that the proposed method converges fast with the increasing sampling points.

In terms of layer numbers $K$, the values are $12,16,20$ and 24 , respectively. Due to the high convergence rate, the displacements of FGPM cylindrical panel are nearly the same when the layer number is bigger than 4 . In this part, only the electric potential results are depicted to analyse. As shown in Fig. 4, the more layer numbers, the smaller value of electric potential is. It is indicted that the proposed method has good convergence, especially the high convergence rate of displacement. However, for the electric potential, the results change little after the $K$ is more than 20 .

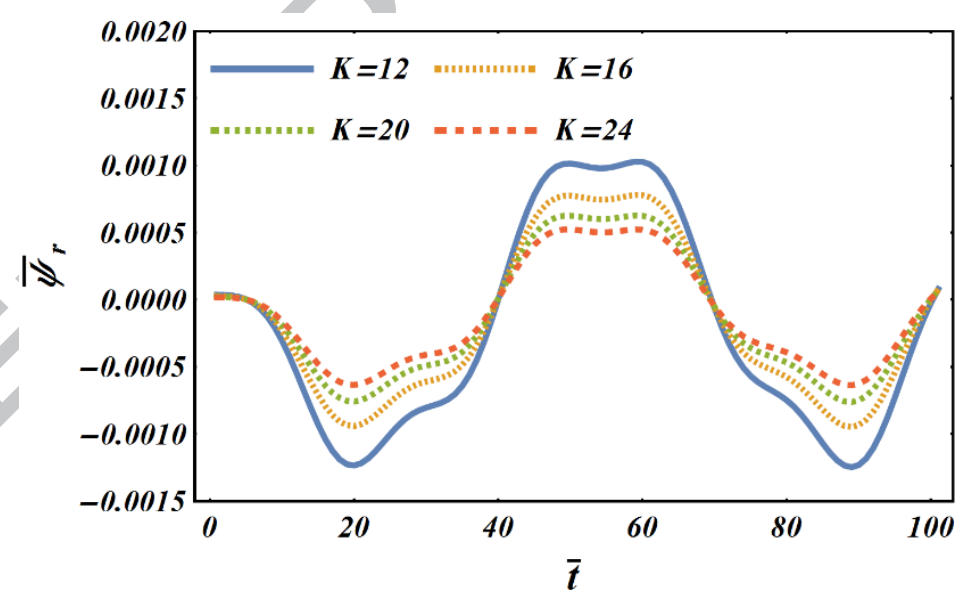

Fig. 4 Electric potential with layer numbers.

\subsection{Effect of initial electric potential}

Under the circumstances of simply supported, the outer surface of the cylindrical panel is electrically grounded to zero potential, while the values of $V_{0, b}$ are $0,0.001,0.005$ and 0.01 , respectively. The relation between the normalised electric potential and time history at the central 
position is shown in Fig. 5(a). When the internal surface and outer surface are applied to initial electric potential as Fig. 5(b), the investigation is also carried out.

(a)

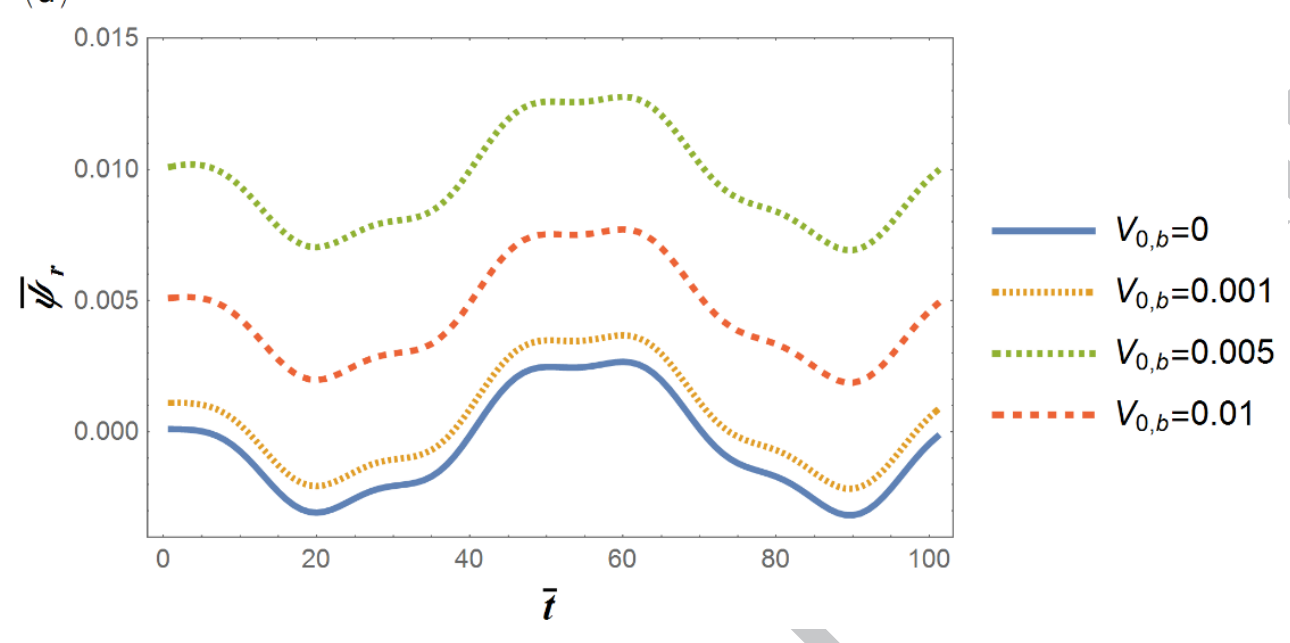

(b)

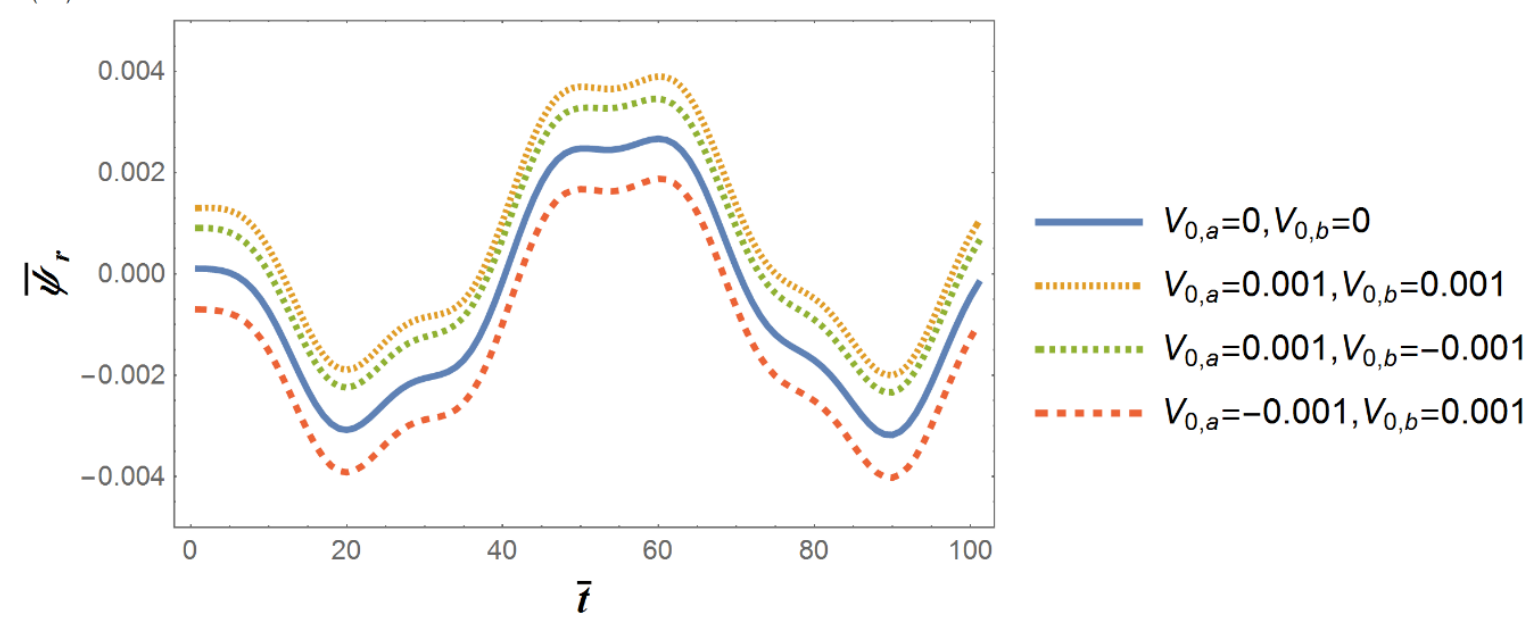

Fig. 5 Electric potential under various initial values.

While the mechanical load is applying on the outer surface, the inner surface has different

values of initial electric potential. As shown in Fig. 5(a), the change of potential at the central position is consistent with the initial potential. When two sides of the surface ( $r=a$ and $r=b$ ) have the same modulus but opposite polarity, the results are depicted in Fig. 5(b). Because the SSM calculates the transfer matrix of the FGPM panel from the outer surface to the bottom surface, the central potential at $t=0$ is the same as the initial electric potential of the outer surface. And there is 
no doubt that the potential at $r=b$ has also affected. When plus and minus signs of the two sides are opposite, the value of central electric potential will decrease. If not, the value will increase.

\subsection{Effect of FG index}

To study the effects of FG index, the deflection and electric potential along the radial direction of FGPM cylindrical panels are examined here. The sampling point number and the layer number are 21 and 4 respectively. Furthermore, the boundary condition used in this section is the same as case 2 in Table. 3. And four different values of FG index $(\gamma=0.2,0.5,2,5)$ are considered.
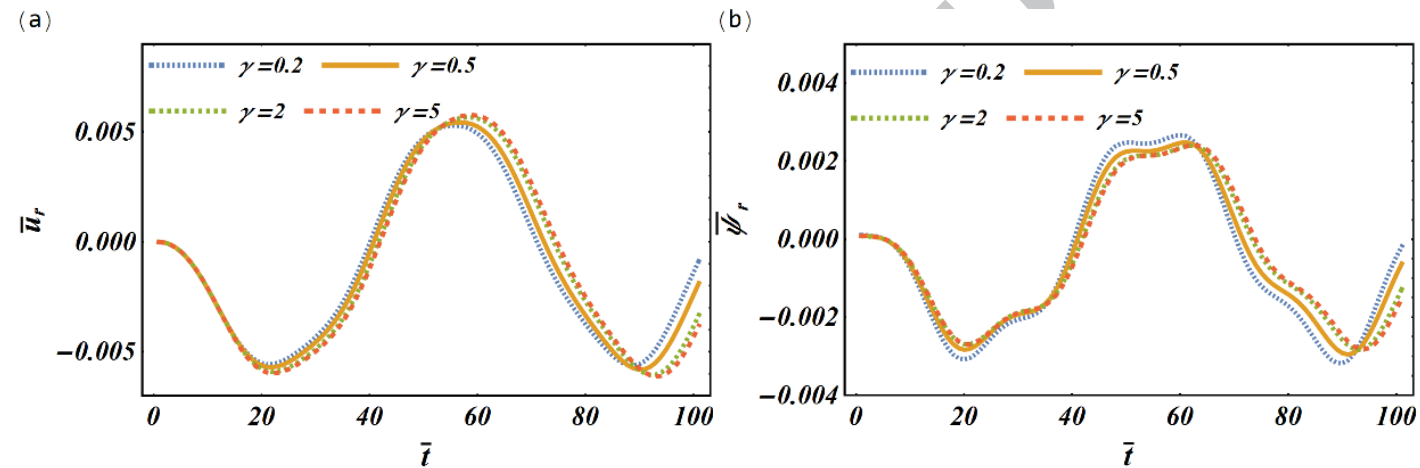

Fig. 6 Deflection and electric potential with different values of variation law.

Power laws with different FG index $\gamma$ show similar trend on the transient response of FGPM cylindrical panels at the central position in Fig. 6. In terms of displacement, the deflection amplitude of the panel increases as the FG index increases. But for the electric potential, the increase of FG index decreases the electric potential amplitude of the FGP panel. However, the influence of this factor is relatively small.

\subsection{Effect of central angle}

In this part, the effect of central angle $\alpha$ has been investigated. Under the circumstances of case 2 , the values of $\alpha$ which are $30^{\circ}, 45^{\circ}, 60^{\circ}$ and $90^{\circ}$ have been carried out. The comparison of normalised displacement is shown in Fig. 7. 


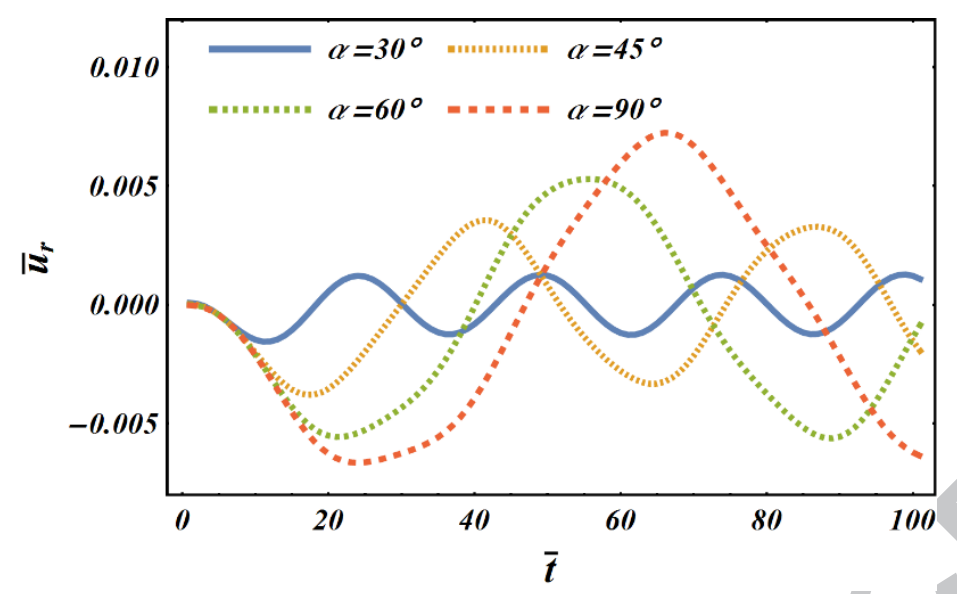

Fig. 7 Deflection history with different central angles.

In certain conditions, different central angles refer to different prestress and resistance.

Generally speaking, applied to the same external force, the deflection increases with the increase of central angle. In Fig. 7, the displacement amplitude along the radial direction becomes larger when the central angle increases, but the period decreases at the same time. Because of quantitative energy input, the vibration amplitude and its period time present a negative correlation.

\section{Conclusion}

In this article, the transient vibration of an FGPM cylindrical panel with various boundary conditions has been investigated. Based on the SSM and DQM, governing equations are derived. In order to obtain the solutions, Laplace transform and its numerical inversion are carried out. According to the results, we can draw the conclusions as below:

-The proposed method has a good agreement with the published literature and ANSYS. That means the method is accurate and semi-analytical solutions are credible.

- The convergence study shows that the proposed method has a high convergence rate. Furthermore, the displacement converges earlier than the electric potential in the time domain.

- The analysis of initial electric potential on the outer surface and bottom surface indicates that 
when the plus and minus signs of two sides are opposite, the value of central electric potential will decrease. If not, the value will increase.

-Under simply supported condition, four different values of FG index are considered. In terms of displacement, the deflection amplitude of the panel increases as FG index $\gamma$ increases. But for the electric potential, the increase of FG index decreases the electric potential amplitude of the FGPM panel.

- The effect of central angle has been analysed here. The amplitude of displacements along the radial direction becomes larger when the central angle increases, but the period time decreases at the same time. Thus, the central angle has a strong correlation with dynamic behaviour.

The paper fills a gap in solving the dynamic problems of FGPM structures by using the semianalytical approach in various boundary conditions. Moreover, the conclusions can be the foundation for future studies in the field of aerospace and intelligent structures.

\section{Acknowledgments}

This work was supported by the National Natural Science Foundation of China (Grant No.

$51879231,51679214,51409228)$.

Appendix A 


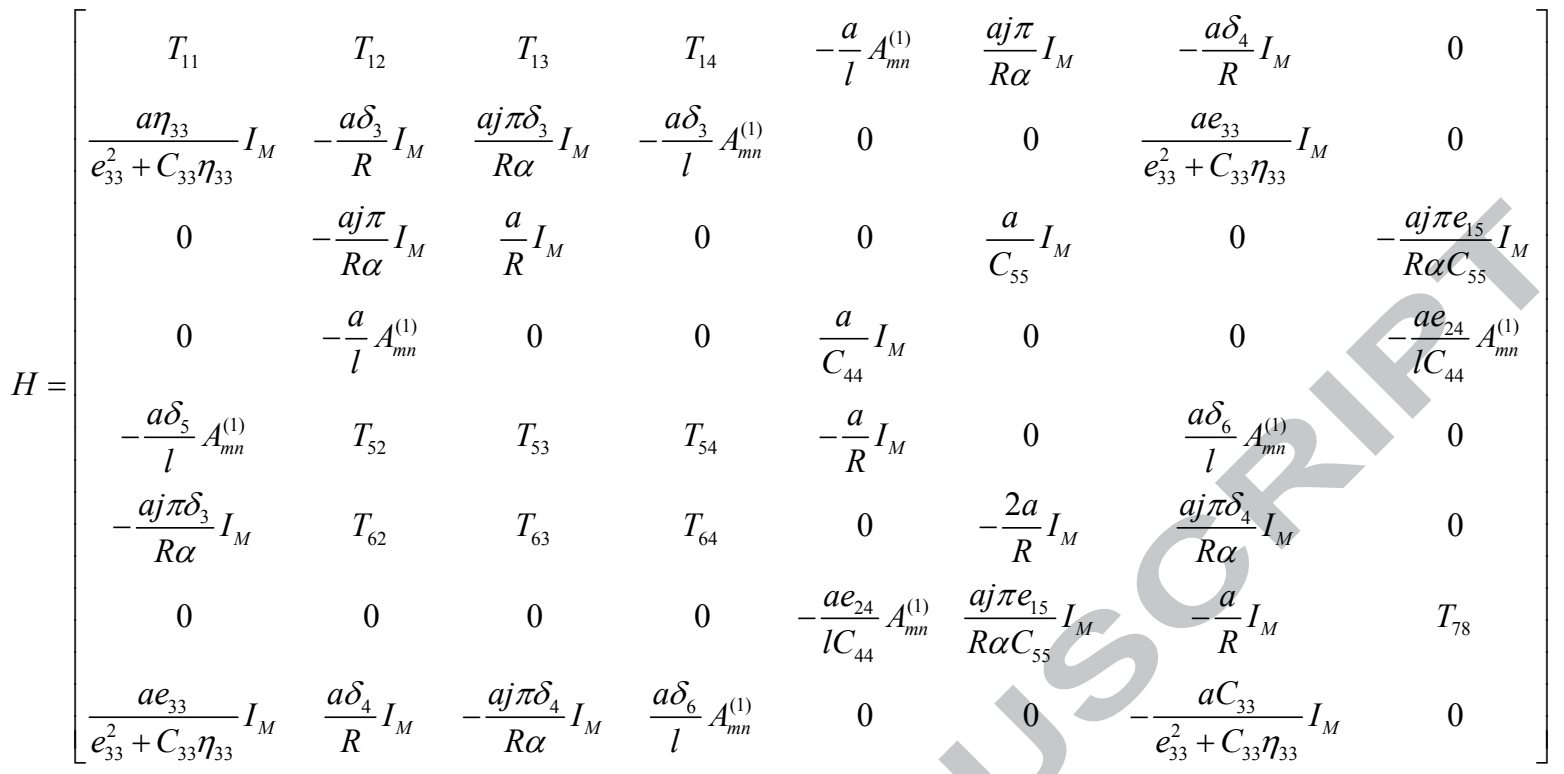

$$
\begin{aligned}
& T_{11}=\frac{a\left(\delta_{1}+\delta_{2}\right)}{R} I_{M} T_{12}=a\left(\rho s^{2}+\frac{C_{33} \delta_{10}-C_{13} \delta_{3}+e_{33} \delta_{9}}{R^{2}}\right) I_{M} \\
& T_{13}=-\frac{a j \pi\left(-C_{13} \delta_{3}+e_{33} \delta_{9}+C_{33} \delta_{10}\right)}{R^{2} \alpha} I_{M} T_{14}=\frac{a\left(-C_{23} \delta_{3}-e_{33} \delta_{7}+C_{33} \delta_{8}\right)}{R l} A_{m n}^{(1)} \\
& T_{52}=\frac{a\left(C_{23} \delta_{3}-C_{33} \delta_{8}+e_{33} \delta_{7}\right)}{R l} A_{m n}^{(1)} \quad T_{53}=\frac{\operatorname{aj} \pi\left(C_{12}+C_{66}-C_{23} \delta_{3}+e_{32} \delta_{4}\right)}{R l \alpha} A_{m n}^{(1)} \\
& T_{54}=a\left(\rho s^{2}+\frac{j^{2} \pi^{2} C_{66}}{R^{2} \alpha^{2}}\right) I_{M}+\frac{a\left(-C_{33}+e_{32} \delta_{6}+C_{23} \delta_{5}\right)}{l^{2}} A_{m n}^{(2)} \\
& T_{62}=-\frac{\operatorname{aj} \pi\left(-C_{13} \delta_{3}+C_{33} \delta_{10}+e_{33} \delta_{9}\right)}{R^{2} \alpha} I_{M} \\
& T_{63}=a\left(\rho s^{2}+\frac{j^{2} \pi^{2}\left(-C_{13} \delta_{3}+e_{33} \delta_{9}+C_{33} \delta_{10}\right)}{R^{2} \alpha^{2}}\right) I_{M}-\frac{a C_{66}}{l^{2}} A_{m n}^{(2)} \\
& T_{64}=-\frac{a j \pi\left(C_{12}+C_{66}-C_{23} \delta_{3}+e_{32} \delta_{4}\right)}{R l \alpha} A_{m n}^{(1)} T_{78}=-\frac{a j^{2} \pi^{2} \delta_{11}\left(e_{33}^{2}+C_{33} \eta_{33}\right)}{R^{2} \alpha^{2} C_{55}} I_{M}+\frac{a \delta_{12}\left(e_{33}^{2}+C_{33} \eta_{33}\right)}{l^{2} C_{44}} A_{m n}^{(2)} \\
& \mathbf{I}_{M}=\left[\begin{array}{cccc}
1 & 0 & \cdots & 0 \\
0 & 1 & \cdots & 0 \\
\vdots & \vdots & \ddots & \vdots \\
0 & 0 & \cdots & 1
\end{array}\right]_{M \times M}, \text { and } \mathbf{A}_{m n}^{(i)}=\left[\begin{array}{cccc}
A_{11}^{(i)} & A_{12}^{(i)} & \cdots & A_{1 M}^{(i)} \\
A_{21}^{(i)} & A_{22}^{(i)} & \cdots & A_{2 M}^{(i)} \\
\vdots & \vdots & \ddots & \vdots \\
A_{M 1}^{(i)} & A_{M 2}^{(i)} & \cdots & A_{M M}^{(i)}
\end{array}\right]
\end{aligned}
$$

Appendix B 


$$
\begin{aligned}
& H=\left[\begin{array}{cccccccc}
H_{11} & H_{12} & H_{13} & H_{14} & H_{15} & \frac{j \pi a I_{2}}{R \alpha} & -\frac{a \delta_{4} I_{2}}{R} & 0 \\
H_{21} & -\frac{a \delta_{3} I_{2}}{R} & \frac{j \pi a \delta_{3} I_{2}}{R \alpha} & H_{24} & 0 & 0 & H_{27} & 0 \\
0 & -\frac{j \pi a I_{2}}{R \alpha} & \frac{a I_{2}}{R} & 0 & 0 & \frac{a I_{2}}{C_{55}} & 0 & H_{38} \\
0 & -\frac{a A_{m n}^{(1)}}{l} & 0 & 0 & \frac{a I_{2}}{C_{44}} & 0 & 0 & H_{48} \\
-\frac{a \delta_{5} A_{m n}^{(1)}}{l} & H_{52} & H_{53} & H_{54} & -\frac{a I_{2}}{R} & 0 & \frac{a \delta_{6} A_{m n}^{(1)}}{l} & 0 \\
-\frac{j \pi a \delta_{3} I_{2}}{R \alpha} & H_{62} & H_{63} & H_{64} & 0 & -\frac{2 a I_{2}}{R} & \frac{j \pi a \delta_{4} I_{2}}{R \alpha} & 0 \\
0 & 0 & 0 & 0 & H_{75} & H_{76} & -\frac{a I_{2}}{R} & H_{78} \\
H_{81} & \frac{a \delta_{4} I_{2}}{R} & -\frac{j \pi a \delta_{4} I_{2}}{R \alpha} & H_{84} & 0 & 0 & H_{87} & 0
\end{array}\right] \\
& H_{11}=\frac{a\left(\delta_{1}+\delta_{2}\right) I_{2}}{R} H_{21}=\frac{a \eta_{33} I_{2}}{e_{33}^{2}+C_{33} \eta_{33}} H_{81}=\frac{a e_{33} I_{2}}{e_{33}^{2}+C_{33} \eta_{33}} \quad H_{12}=\left(s^{2} \rho a+\frac{-a C_{13} \delta_{3}+a e_{33} \delta_{9}+a C_{33} \delta_{10}}{R^{2}}\right) I_{2} \\
& H_{52}=\frac{a\left(C_{23} \delta_{3}+e_{33} \delta_{7}-C_{33} \delta_{8}\right) A_{m n}^{(1)}}{l R} \quad H_{13}=H_{62}=\frac{j \pi a\left(C_{13} \delta_{3}-e_{33} \delta_{9}-C_{33} \delta_{10}\right) I_{2}}{R^{2} \alpha} \\
& H_{53}=\frac{j \pi a\left(C_{12}+C_{66}-C_{23} \delta_{3}+e_{32} \delta_{4}\right) A_{m n}^{(1)}}{l R \alpha} \quad H_{63}=\left(a \rho s^{2}+\frac{j^{2} \pi^{2} a\left(-C_{13} \delta_{3}+e_{33} \delta_{9}+C_{33} \delta_{10}\right)}{R^{2} \alpha^{2}}\right) I_{2}-\frac{a C_{66} A_{m n}^{(2)}}{l^{2}} \\
& H_{14}=\frac{a\left(C_{23} \delta_{3}+e_{33} \delta_{7}-C_{33} \delta_{8}\right) A_{1 n}^{(1)}\left(A_{M 1}^{(1)} A_{m M}^{(1)}-A_{m 1}^{(1)} A_{M M}^{(1)}\right)}{l R\left(A_{1 M}^{(1)} A_{M 1}^{(1)}-A_{11}^{(1)} A_{M M}^{(1)}\right)}-\frac{a\left(C_{23} \delta_{3}+e_{33} \delta_{7}-C_{33} \delta_{8}\right)}{l R}\left(A_{m n}^{(1)}-\frac{A_{1 M}^{(1)} A_{m 1}^{(1)} A_{M n}^{(1)}-A_{11}^{(1)} A_{m M}^{(1)} A_{M n}^{(1)}}{A_{1 M}^{(1)} A_{M 1}^{(1)}-A_{11}^{(1)} A_{M M}^{(1)}}\right) \\
& H_{24}=\frac{a \delta_{5} A_{1 n}^{(1)}\left(A_{M 1}^{(1)} A_{m M}^{(1)}-A_{m 1}^{(1)} A_{M M}^{(1)}\right)}{l\left(A_{1 M}^{(1)} A_{M 1}^{(1)}-A_{11}^{(1)} A_{M M}^{(1)}\right)}-\frac{a \delta_{5}}{l}\left(A_{m n}^{(1)}-\frac{A_{1 M}^{(1)} A_{m}^{(1)} A_{M n}^{(1)}-A_{11}^{(1)} A_{m M}^{(1)} A_{M n}^{(1)}}{A_{1 M}^{(1)} A_{M 1}^{(1)}-A_{11}^{(1)} A_{M M}^{(1)}}\right) \\
& H_{54}=\left(a \rho s^{2}+\frac{j^{2} \pi^{2} a C_{66}}{R^{2} \alpha^{2}}\right) I_{2}+\frac{a\left(C_{33}-C_{23} \delta_{5}-e_{32} \delta_{6}\right) A_{1 n}^{(1)}\left(A_{M 1}^{(1)} A_{m M}^{(2)}-A_{m 1}^{(2)} A_{M M}^{(1)}\right)}{l^{2}\left(A_{1 M}^{(1)} A_{M 1}^{(1)}-A_{11}^{(1)} A_{M M}^{(1)}\right)} \\
& -\frac{a\left(C_{33}-C_{23} \delta_{5}-e_{32} \delta_{6}\right)}{l^{2}}\left(A_{m n}^{(2)}-\frac{A_{1 M}^{(1)} A_{m 1}^{(2)} A_{M n}^{(1)}-A_{11}^{(1)} A_{m M}^{(2)} A_{M n}^{(1)}}{A_{1 M}^{(1)} A_{M 1}^{(1)}-A_{11}^{(1)} A_{M M}^{(1)}}\right) \\
& H_{64}=\frac{j \pi a\left(C_{12}+C_{66}-C_{23} \delta_{3}+e_{32} \delta_{4}\right) A_{1 n}^{(1)}\left(A_{M 1}^{(1)} A_{m M}^{(1)}-A_{m 1}^{(1)} A_{M M}^{(1)}\right)}{\operatorname{lR} \alpha\left(A_{1 M}^{(1)} A_{M 1}^{(1)}-A_{11}^{(1)} A_{M M}^{(1)}\right)} \\
& -\frac{j \pi a\left(C_{12}+C_{66}-C_{23} \delta_{3}+e_{32} \delta_{4}\right)}{l R \alpha}\left(A_{m n}^{(1)}-\frac{A_{1 M}^{(1)} A_{m 1}^{(1)} A_{M n}^{(1)}-A_{11}^{(1)} A_{m M}^{(1)} A_{M n}^{(1)}}{A_{1 M}^{(1)} A_{M 1}^{(1)}-A_{11}^{(1)} A_{M M}^{(1)}}\right) \\
& H_{84}=-\frac{a \delta_{6} A_{1 n}^{(1)}\left(A_{M 1}^{(1)} A_{m M}^{(1)}-A_{m 1}^{(1)} A_{M M}^{(1)}\right)}{l\left(A_{1 M}^{(1)} A_{M 1}^{(1)}-A_{11}^{(1)} A_{M M}^{(1)}\right)}+\frac{a \delta_{6}}{l}\left(A_{m n}^{(1)}-\frac{A_{1 M}^{(1)} A_{m 1}^{(1)} A_{M n}^{(1)}-A_{11}^{(1)} A_{m M}^{(1)} A_{M n}^{(1)}}{A_{1 M}^{(1)} A_{M 1}^{(1)}-A_{11}^{(1)} A_{M M}^{(1)}}\right) \\
& H_{15}=-\frac{a\left(A_{m 1}^{(1)}+A_{m M}^{(1)}+A_{m n}^{(1)}\right)}{l} \quad H_{75}=-\frac{a e_{24}\left(A_{m 1}^{(1)}+A_{m M}^{(1)}+A_{m n}^{(1)}\right)}{l C_{44}} \quad H_{76}=\frac{j \pi a e_{15} I_{2}}{R \alpha C_{55}}
\end{aligned}
$$




$$
\begin{aligned}
& H_{27}=\frac{a e_{33} I_{2}}{e_{33}^{2}+C_{33} \eta_{33}} H_{87}=-\frac{a C_{33} I_{2}}{e_{33}^{2}+C_{33} \eta_{33}} H_{38}=-\frac{j \pi a e_{15} I_{2}}{R \alpha C_{55}} H_{48}=-\frac{e_{24} a A_{m n}^{(1)}}{l C_{44}} \\
& H_{78}=-\frac{j^{2} \pi^{2} a \delta_{11}\left(e_{33}^{2}+C_{33} \eta_{33}\right) I_{2}}{R^{2} \alpha^{2} C_{55}}+\frac{a\left(e_{33}^{2}+C_{33} \eta_{33}\right) \delta_{12} A_{m n}^{(2)}}{l^{2} C_{44}} \\
& \mathrm{C}-\mathrm{C} \\
& H=\left[\begin{array}{cccccccc}
H_{11} & H_{12} & H_{13} & H_{14} & -\frac{a A_{m n}^{(1)}}{l} & \frac{j \pi a I_{2}}{R \alpha} & -\frac{a \delta_{4} I_{2}}{R} & H_{18} \\
H_{21} & -\frac{a \delta_{3} I_{2}}{R} & \frac{j \pi a \delta_{3} I_{2}}{R \alpha} & H_{24} & 0 & 0 & H_{27} & 0 \\
0 & -\frac{j \pi a I_{2}}{R \alpha} & \frac{a I_{2}}{R} & 0 & 0 & \frac{a I_{2}}{C_{55}} & 0 & H_{38} \\
0 & -\frac{a A_{m n}^{(1)}}{l} & 0 & 0 & \frac{a I_{2}}{C_{44}} & 0 & 0 & H_{48} \\
-\frac{a \delta_{5} A_{m n}^{(1)}}{l} & H_{52} & H_{53} & H_{54} & -\frac{a I_{2}}{R} & 0 & \frac{a \delta_{6} A_{m n}^{(1)}}{l} & 0 \\
-\frac{j \pi a \delta_{3} I_{2}}{R \alpha} & H_{62} & H_{63} & H_{64} & 0 & -\frac{2 a I_{2}}{R} & \frac{j \pi a \delta_{4} I_{2}}{R \alpha} & 0 \\
0 & H_{72} & 0 & 0 & H_{75} & H_{76} & -\frac{a I_{2}}{R} & H_{78} \\
H_{81} & \frac{a \delta_{4} I_{2}}{R} & -\frac{j \pi a \delta_{4} I_{2}}{R \alpha} & \frac{a \delta_{6} A_{m n}^{(1)}}{l} & 0 & 0 & H_{87} & 0
\end{array}\right]
\end{aligned}
$$$$
H_{11}=\frac{a\left(\delta_{1}+\delta_{2}\right) I_{2}}{R} H_{21}=\frac{a \eta_{33} I_{2}}{e_{33}^{2}+C_{33} \eta_{33}} H_{81}=\frac{e_{33} a I_{2}}{e_{33}^{2}+C_{33} \eta_{33}}
$$$$
H_{12}=\left(a \rho s^{2}+\frac{-a C_{13} \delta_{3}+a e_{33} \delta_{9}+a C_{33} \delta_{10}}{R^{2}}\right) I_{2}-\frac{a C_{44}}{l^{2}}\left(A_{1 n}^{(1)} A_{m 1}^{(1)}+A_{m M}^{(1)} A_{M n}^{(1)}\right)
$$$$
H_{52}=\frac{a\left(C_{23} \delta_{3}+e_{33} \delta_{7}-C_{33} \delta_{8}\right) A_{m n}^{(1)}}{l R} H_{13}=H_{62}=\frac{j \pi a\left(C_{13} \delta_{3}-e_{33} \delta_{9}-C_{33} \delta_{10}\right) I_{2}}{R^{2} \alpha}
$$$$
H_{72}=-\frac{e_{24} a}{l^{2}}\left(A_{1 n}^{(1)} A_{m 1}^{(1)}+A_{m M}^{(1)} A_{M n}^{(1)}\right) H_{53}=\frac{j \pi a\left(C_{12}+C_{66}-C_{23} \delta_{3}+e_{32} \delta_{4}\right) A_{m n}^{(1)}}{l R \alpha}
$$$$
H_{63}=\left(a \rho s^{2}+\frac{j^{2} \pi^{2} a\left(-C_{13} \delta_{3}+e_{33} \delta_{9}+C_{33} \delta_{10}\right)}{R^{2} \alpha^{2}}\right) I_{2}-\frac{a C_{66} A_{m n}^{(2)}}{l^{2}} H_{14}=-\frac{a\left(C_{23} \delta_{3}+e_{33} \delta_{7}-C_{33} \delta_{8}\right) A_{m n}^{(1)}}{l R}
$$$$
H_{54}=\left(a \rho s^{2}+\frac{j^{2} \pi^{2} a C_{66}}{R^{2} \alpha^{2}}\right) I_{2}-\frac{a}{l^{2}}\left[\left(C_{33} \delta_{5}^{2}-2 e_{33} \delta_{5} \delta_{6}-\eta_{33} \delta_{6}^{2}\right) A_{1 n}^{(1)} A_{m 1}^{(1)}\right.
$$$$
\left.+\left(C_{33}-C_{23} \delta_{5}-e_{32} \delta_{6}\right) A_{m n}^{(2)}\right]
$$$$
H_{64}=-\frac{j \pi a\left(C_{12}+C_{66}-C_{23} \delta_{3}+e_{32} \delta_{4}\right) A_{m n}^{(1)}}{l R \alpha} H_{75}=-\frac{e_{24} a A_{m n}^{(1)}}{l C_{44}} H_{76}=\frac{e_{15} j \pi a I_{2}}{R \alpha C_{55}} H_{27}=\frac{e_{33} a I_{2}}{e_{33}^{2}+C_{33} \eta_{33}}
$$$$
H_{87}=-\frac{a C_{33} I_{2}}{e_{33}^{2}+C_{33} \eta_{33}} \quad H_{18}=-\frac{e_{24} a A_{1 n}^{(1)} A_{m 1}^{(1)}}{l^{2}}-\frac{e_{24} a A_{m M}^{(1)} A_{M n}^{(1)}}{l^{2}} \quad H_{38}=-\frac{e_{15} j \pi a I_{2}}{R \alpha C_{55}}
$$ 


$$
H_{48}=-\frac{e_{24} a A_{m n}^{(1)}}{l C_{44}} \quad H_{78}=-\frac{j^{2} \pi^{2} a \delta_{11}\left(e_{33}^{2}+C_{33} \eta_{33}\right) I_{2}}{R^{2} \alpha^{2} C_{55}}+\frac{a\left(e_{33}^{2}+C_{33} \eta_{33}\right) \delta_{12} A_{m n}^{(2)}}{l^{2} C_{44}}-\frac{e_{24}^{2} a\left(A_{1 n}^{(1)} A_{m 1}^{(1)}+A_{m M}^{(1)} A_{M n}^{(1)}\right)}{l^{2} C_{44}}
$$

C-S

$$
\begin{aligned}
& H=\left[\begin{array}{cccccccc}
H_{11} & H_{12} & H_{13} & H_{14} & H_{15} & \frac{j \pi a I_{2}}{R \alpha} & -\frac{a \delta_{4} I_{2}}{R} & H_{18} \\
H_{21} & -\frac{a \delta_{3} I_{2}}{R} & \frac{j \pi a \delta_{3} I_{2}}{R \alpha} & H_{24} & 0 & 0 & H_{27} & 0 \\
0 & -\frac{j \pi a I_{2}}{R \alpha} & \frac{a I_{2}}{R} & 0 & 0 & \frac{a I_{2}}{C_{55}} & 0 & H_{38} \\
0 & -\frac{a A_{m n}^{(1)}}{l} & 0 & 0 & \frac{a I_{2}}{C_{44}} & 0 & 0 & H_{48} \\
-\frac{a \delta_{5} A_{m n}^{(1)}}{l} & H_{52} & H_{53} & H_{54} & -\frac{a I_{2}}{R} & 0 & \frac{a \delta_{6} A_{m n}^{(1)}}{l} & 0 \\
-\frac{j \pi a \delta_{3} I_{2}}{R \alpha} & H_{62} & H_{63} & H_{64} & 0 & -\frac{2 a I_{2}}{R} & \frac{j \pi a \delta_{4} I_{2}}{R \alpha} & 0 \\
0 & H_{72} & 0 & 0 & H_{75} & H_{76} & -\frac{a I_{2}}{R} & H_{78} \\
H_{81} & \frac{a \delta_{4} I_{2}}{R} & -\frac{j \pi a \delta_{4} I_{2}}{R \alpha} & H_{84} & 0 & 0 & H_{87} & 0
\end{array}\right] \\
& H_{11}=\frac{a\left(\delta_{1}+\delta_{2}\right) I_{2}}{R} H_{21}=\frac{a \eta_{33} I_{2}}{e_{33}^{2}+C_{33} \eta_{33}} H_{81}=\frac{e_{33} a I_{2}}{e_{33}^{2}+C_{33} \eta_{33}} \\
& H_{12}=\left(a \rho s^{2}+\frac{-a C_{13} \delta_{3}+a e_{33} \delta_{9}+a C_{33} \delta_{10}}{R^{2}}\right) I_{2}-\frac{a C_{44} A_{m M}^{(1)} A_{M n}^{(1)}}{l^{2}} H_{52}=\frac{a\left(C_{23} \delta_{3}+e_{33} \delta_{7}-C_{33} \delta_{8}\right) A_{m n}^{(1)}}{l R} \\
& H_{13}=H_{62}=\frac{j \pi a\left(C_{13} \delta_{3}-e_{33} \delta_{9}-C_{33} \delta_{10}\right) I_{2}}{R^{2} \alpha} H_{72}=-\frac{e_{24} a}{l^{2}} A_{m M}^{(1)} A_{M n}^{(1)} \\
& H_{53}=\frac{j \pi a\left(C_{12}+C_{66}-C_{23} \delta_{3}+e_{32} \delta_{4}\right) A_{m n}^{(1)}}{l R \alpha} H_{63}=\left(a \rho s^{2}+\frac{j^{2} \pi^{2} a\left(-C_{13} \delta_{3}+e_{33} \delta_{9}+C_{33} \delta_{10}\right)}{R^{2} \alpha^{2}}\right) I_{2}-\frac{a C_{66} A_{m n}^{(2)}}{l^{2}} \\
& H_{14}=-\frac{a\left(C_{23} \delta_{3}+e_{33} \delta_{7}-C_{33} \delta_{8}\right) A_{11}^{(1)} A_{1 n}^{(1)} A_{m 1}^{(1)}}{l R}-\frac{a\left(C_{23} \delta_{3}+e_{33} \delta_{7}-C_{33} \delta_{8}\right) A_{m n}^{(1)}}{l R} \\
& H_{24}=\frac{a \delta_{5} A_{11}^{(1)} A_{1 n}^{(1)} A_{m 1}^{(1)}}{l}-\frac{a \delta_{5} A_{m n}^{(1)}}{l} \\
& H_{54}=\left(a \rho s^{2}+\frac{j^{2} \pi^{2} a C_{66}}{R^{2} \alpha^{2}}\right) I_{2}+\frac{a\left(C_{33}-C_{23} \delta_{5}-e_{32} \delta_{6}\right) A_{11}^{(1)} A_{1 n}^{(1)} A_{m 1}^{(2)}}{l^{2}} \\
& -\frac{1}{l^{2}} a A_{11}^{(1)}\left[\begin{array}{l}
\left(-C_{33} \delta_{5}^{2}+2 e_{33} \delta_{5} \delta_{6}+\eta_{33} \delta_{6}^{2}\right) A_{1 n}^{(1)} A_{M 1}^{(1)} A_{m M}^{(1)}+\left(C_{33}-C_{23} \delta_{5}-e_{32} \delta_{6}\right) A_{11}^{(1)} A_{m n}^{(2)} \\
+\left(C_{33} \delta_{5}^{2}-2 e_{33} \delta_{5} \delta_{6}-\eta_{33} \delta_{6}^{2}\right) A_{11}^{(1)} A_{m M}^{(1)} A_{M n}^{(1)}
\end{array}\right] \\
& H_{64}=\frac{j \pi a\left(C_{12}+C_{66}-C_{23} \delta_{3}+e_{32} \delta_{4}\right) A_{11}^{(1)} A_{1 n}^{(1)} A_{m 1}^{(1)}}{l R \alpha}-\frac{j \pi a\left(C_{12}+C_{66}-C_{23} \delta_{3}+e_{32} \delta_{4}\right) A_{m n}^{(1)}}{l R \alpha} \\
& H_{84}=-\frac{a \delta_{6} A_{11}^{(1)} A_{1 n}^{(1)} A_{m 1}^{(1)}}{l}+\frac{a \delta_{6} A_{m n}^{(1)}}{l} \quad H_{15}=-\frac{a}{l}\left(A_{m 1}^{(1)}+A_{m n}^{(1)}\right) \quad H_{75}=-\frac{e_{24} a}{l C_{44}}\left(A_{m 1}^{(1)}+A_{m n}^{(1)}\right)
\end{aligned}
$$




$$
\begin{aligned}
& H_{76}=\frac{e_{15} j \pi a I_{2}}{R \alpha C_{55}} \quad H_{27}=\frac{e_{33} a I_{2}}{e_{33}^{2}+C_{33} \eta_{33}} \quad H_{87}=-\frac{a C_{33} I_{2}}{e_{33}^{2}+C_{33} \eta_{33}} \quad H_{18}=-\frac{e_{24} a A_{m M}^{(1)} A_{M n}^{(1)}}{l^{2}} \\
& H_{38}=-\frac{e_{15} j \pi a I_{2}}{R \alpha C_{55}} \quad H_{48}=-\frac{e_{24} a A_{m n}^{(1)}}{l C_{44}} \quad H_{78}=-\frac{j^{2} \pi^{2} a \delta_{11}\left(e_{33}^{2}+C_{33} \eta_{33}\right) I_{2}}{R^{2} \alpha^{2} C_{55}}+\frac{a\left(e_{33}^{2}+C_{33} \eta_{33}\right) \delta_{12} A_{m n}^{(2)}}{l^{2} C_{44}}-\frac{e_{24}^{2} a A_{m M}^{(1)} A_{M n}^{(1)}}{l^{2} C_{44}}
\end{aligned}
$$

\section{References:}

[1] Rajasekaran S, Bakhshi Khaniki H. Finite element static and dynamic analysis of axially functionally graded nonuniform small-scale beams based on nonlocal strain gradient theory. Mech Adv Mater Struc 2018:1-15.

[2] Fu Y, Yao J, Wan Z, Zhao G. Free vibration analysis of moderately thick orthotropic functionally graded plates with general boundary restraints. Mater 2018;11.

[3] Yazdani Sarvestani H, Akbarzadeh AH, Mirabolghasemi A. Structural analysis of size-dependent functionally graded doubly-curved panels with engineered microarchitectures. Acta Mech 2018;229:2675-701.

[4] Selim BA, Zhang LW, Liew KM. Active vibration control of FGM plates with piezoelectric layers based on Reddy' s higher-order shear deformation theory. Compos Struct 2016;155:118-34.

[5] Wang W, Li P, Jin F, Wang J. Vibration analysis of piezoelectric ceramic circular nanoplates considering surface and nonlocal effects. Compos Struct 2016;140:758-75.

[6] Selim BA, Zhang LW, Liew KM. Active vibration control of CNT-reinforced composite plates with piezoelectric layers based on Reddy' s higher-order shear deformation theory. Compos Struct 2017;163:350-64.

[7] Vel SS, Batra RC. Cylindrical Bending of Laminated Plates with Distributed and Segmented Piezoelectric Actuators/Sensors. Aiaa J 2012;38:857-67.

[8] Kumar A, Sharma A, Vaish R, Kumar R, Jain SC. A numerical study on anomalous behavior of piezoelectric response in functionally graded materials. J Mater Sci 2018;53:2413-23.

[9] Mallik N, Ray MC. Effective Coefficients of Piezoelectric Fiber-Reinforced Composites. Aiaa J 2015;41:704-10.

[10] Panda S, Ray MC. Control of Nonlinear Vibrations of Functionally Graded Plates Using 1-3 Piezoelectric Composite. Aiaa J 2015;47:1421-34.

[11] Nguyen LB, Thai CH, Zenkour AM, Nguyen-Xuan H. An isogeometric Bézier finite element method for vibration analysis of functionally graded piezoelectric material porous plates. Int J Mech Sci 2019;157-158:165-83.

[12] Mallek H, Jrad H, Algahtani A, Wali M, Dammak F. Geometrically non-linear analysis of FGCNTRC shell structures with surface-bonded piezoelectric layers. Comput Method Appl M 2019;347:679-99.

[13] Singh AK, Koley S, Negi A, Ray A. On the dynamic behavior of a functionally graded viscoelasticpiezoelectric composite substrate subjected to a moving line load. Eur Phys J Plus 2019;134.

[14] Lian Y, He X, Shi S, Li X, Yang Z, Sun J. A multi-parameter perturbation solution for functionally graded piezoelectric cantilever beams under combined loads. Mater 2018;11.

[15] Mikaeeli S, Behjat B. Three-dimensional analysis of thick functionally graded piezoelectric plate using EFG method. Compos Struct 2016;154:591-9.

[16] Jandaghian AA, Rahmani O. Vibration analysis of functionally graded piezoelectric nanoscale plates 
by nonlocal elasticity theory: An analytical solution. Superlattice Microst 2016;100:57-75.

[17] Wu XH, Chen C, Shen YP, Tian XG. A high order theory for functionally graded piezoelectric shells. Int J Solids Struct 2002;39:5325-44.

[18] Mallek H, Jrad H, Wali M, Dammak F. Piezoelastic response of smart functionally graded structure with integrated piezoelectric layers using discrete double directors shell element. Compos Struct 2019;210:354-66.

[19] Atai AA, Lak D. Analytic investigation of effect of electric field on elasto-plastic response of a functionally graded piezoelectric hollow sphere. J Mech Sci Technol 2016;30:113-9.

[20] Su Z, Jin G, Ye T. Vibration analysis and transient response of a functionally graded piezoelectric curved beam with general boundary conditions. Smart Mater Struct 2016;25.

[21] Ma Y, He T. The transient response of a functionally graded piezoelectric rod subjected to a moving heat source under fractional order theory of thermoelasticity. Mech Adv Mater Struc 2017;24:78996.

[22] Zhu S, Jin G, Ye T. Electro-mechanical vibration characteristics of functionally graded piezoelectric plates with general boundary conditions. Int J Mech Sci 2018;s 138 - 139:42-53.

[23] Bodaghi M, Shakeri M. An analytical approach for free vibration and transient response of functionally graded piezoelectric cylindrical panels subjected to impulsive loads. Compos Struct 2012;94:1721-35.

[24] Mehralian F, Beni YT. Vibration analysis of size-dependent bimorph functionally graded piezoelectric cylindrical shell based on nonlocal strain gradient theory. J Braz Soc Mech Sci 2018;40:27.

[25] Fan J, Ye J. An exact solution for the statics and dynamics of laminated thick plates with orthotropic layers. Int J Solids Struct 1990;26:655-62.

[26] Li Y, Shi Z. Free vibration of a functionally graded piezoelectric beam via state-space based differential quadrature. Compos Struct 2009;87:257-64.

[27] Jodaei A, Jalal M, Yas MH. Three-dimensional free vibration analysis of functionally graded piezoelectric annular plates via SSDQM and comparative modeling by ANN. Math Comput Modelling 2013;57:1408-25.

[28] Chen WQ, Bian ZG, Lv CF, Ding HJ. 3D free vibration analysis of a functionally graded piezoelectric hollow cylinder filled with compressible fluid. Int J Solids Struct 2004;41:947-64.

[29] Malekzadeh P, Ghaedsharaf M. Three-dimensional thermoelastic analysis of finite length laminated cylindrical panels with functionally graded layers. Meccanica 2014;49:887-906.

[30] Nie GJ, Zhong Z. Semi-analytical solution for three-dimensional vibration of functionally graded circular plates. Comput Method Appl M 2007;196:4901-10.

[31] Lü CF, Zhang ZC, Chen WQ. Free vibration of generally supported rectangular Kirchhoff plates: State - space - based differential quadrature method. Int J Numer Meth Eng 2010;70:1430-50.

[32] JODAEI, JALAL, M. H. Free vibration analysis of functionally graded annular plates by state-space based differential quadrature method and comparative modeling by ANN. Compos Part B 2012;43:340-53.

[33] Chen WQ, Lv CF, Bian ZG. Elasticity solution for free vibration of laminated beams. Compos Struct 2003;62:75-82.

[34] Xu L, Wang Z, Wang L, Liu G. Semi-analytical solution for three-dimensional transient response of functionally graded annular plate on a two parameter viscoelastic foundation. J Sound Vib 2014;333:2649-63. 
[35] Cohen, Alan M. Numerical Methods for Laplace Transform Inversion. Springer Berlin 2007;5.

[36] Wang Z, Xu L, Fallah AS, Liu G, Louca LA, Wang L. A novel efficient method to evaluate the dynamic response of laminated plates subjected to underwater shock. J Sound Vib 2013;332:561834.

[37] Durbin F. Numerical Inversion of Laplace Transforms: An Efficient Improvement to Dubner and Abate's Method. Comput J 1972;17:371-6.

[38] Xu L, Kou HL, Wang L, Palmer AC, Wang Z, Liu G. Three-dimensional transient analysis of functionally graded material annular sector plate under various boundary conditions. Compos Struct 2015;132:584-96.

[39] Bodaghi M, Shakeri M. An analytical approach for free vibration and transient response of functionally graded piezoelectric cylindrical panels subjected to impulsive loads. Compos Struct 2012;94:1721-35.

[40] Yang J.Special Topics in the Theory of Piezoelectricity2009.

[41] Liang X, Kou HL, Liu GH, Wang LZ, Wang ZY, Wu ZJ. A semi-analytical state-space approach for $3 \mathrm{D}$ transient analysis of functionally graded material cylindrical shells. J Zhejiang Univ: Sci A 2015;16:525-40.

[42] Liang X, Wu Z, Wang L, Liu G, Wang Z, Zhang W. Semianalytical Three-Dimensional Solutions for the Transient Response of Functionally Graded Material Rectangular Plates. J Eng Mech 2014; 141. 


\section{List of tables}

Table.1 Fundamental material properties along the radial direction.

Table.2 Natural frequencies of the first mode for FGPM cylindrical panels.

Table. 3 The cases with different variables and boundaries. 
Table. 4 Fundamental material properties along the radial direction.

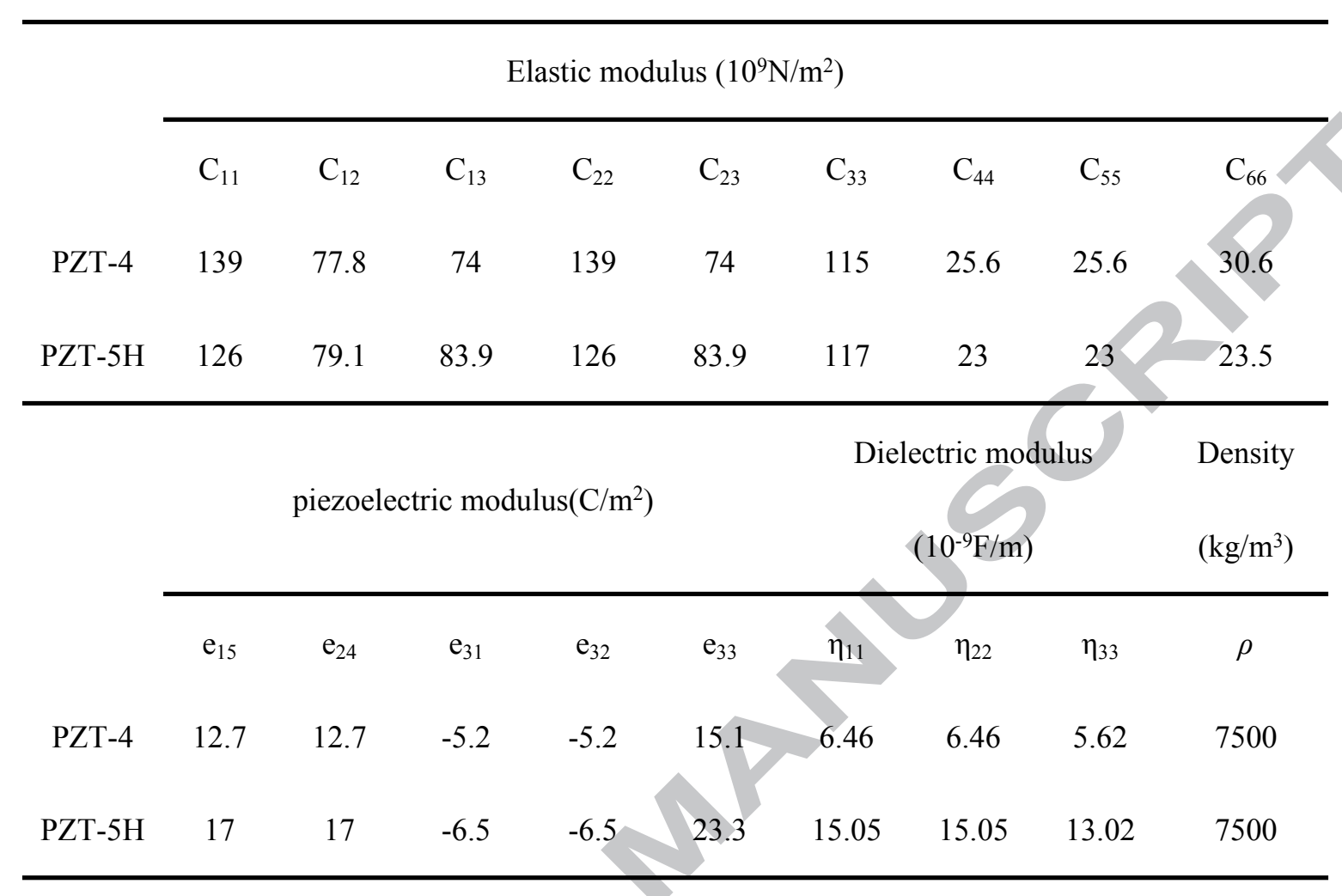


Table. 5 Natural frequencies of the first mode for FGPM cylindrical panels.

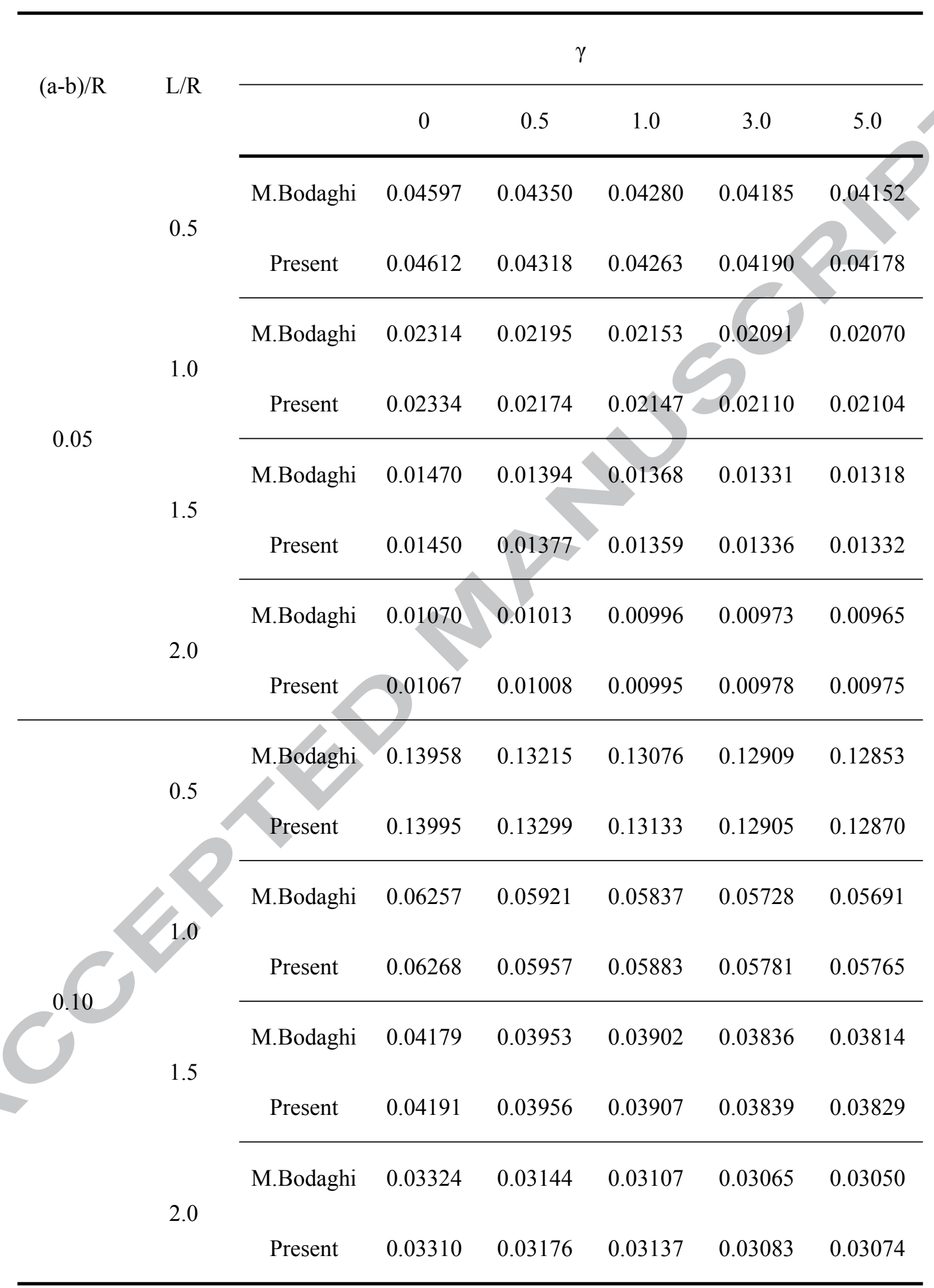


Table. 6 The cases with different variables and boundaries.

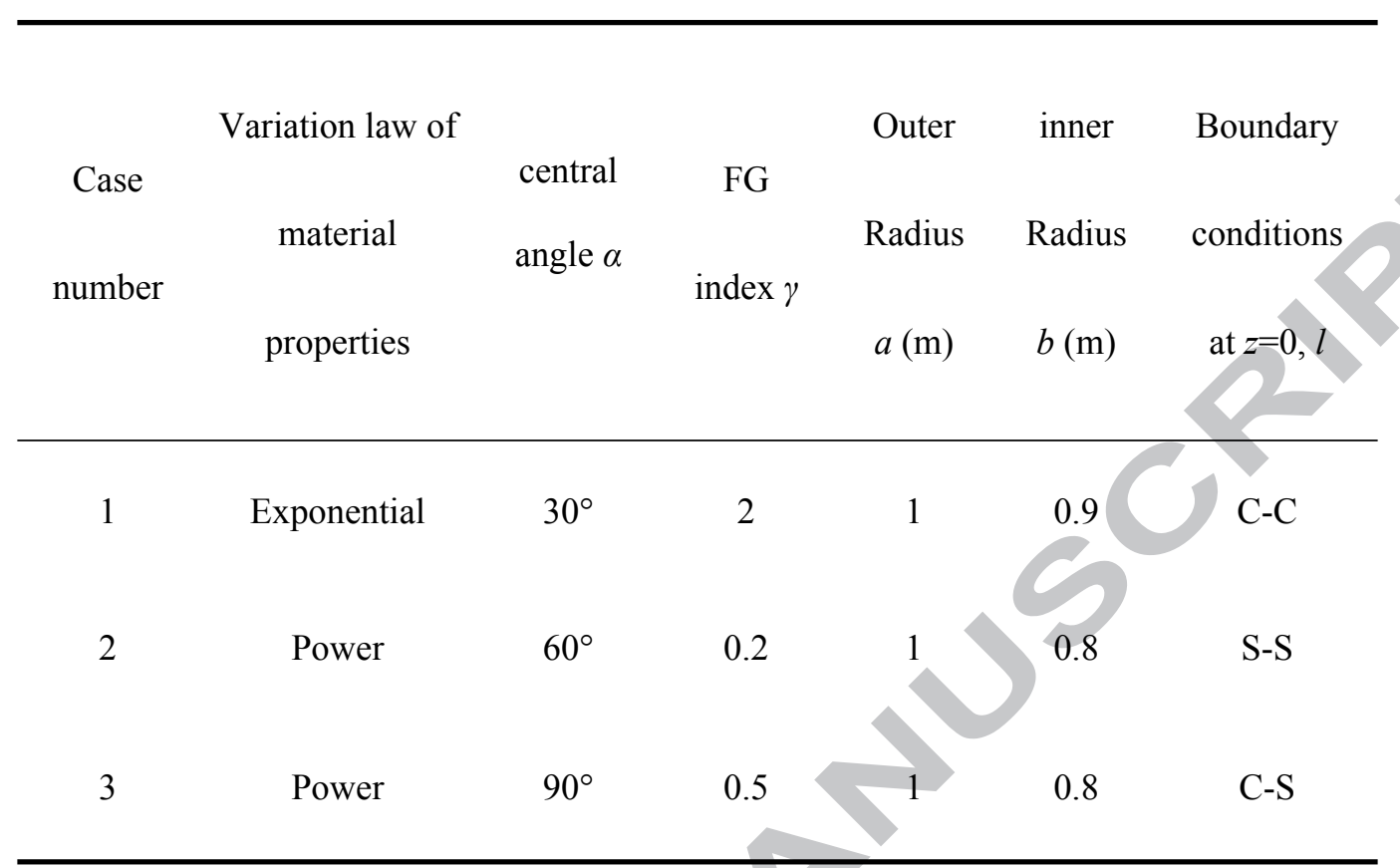




\section{List of figures}

Fig.1 The FGPM cylindrical panel and its cross section.

Fig.2 Deflection and electric potential (a,b) case 1; (c,d) case 2;(e,f) case 3.

Fig.3 Deflection and electric potential with sampling points.

Fig.4 Electric potential with layer numbers.

Fig.5 Electric potential under various initial values.

Fig.6 Deflection and electric potential with different values of variation law.

Fig.7 Deflection history with different central angles. 


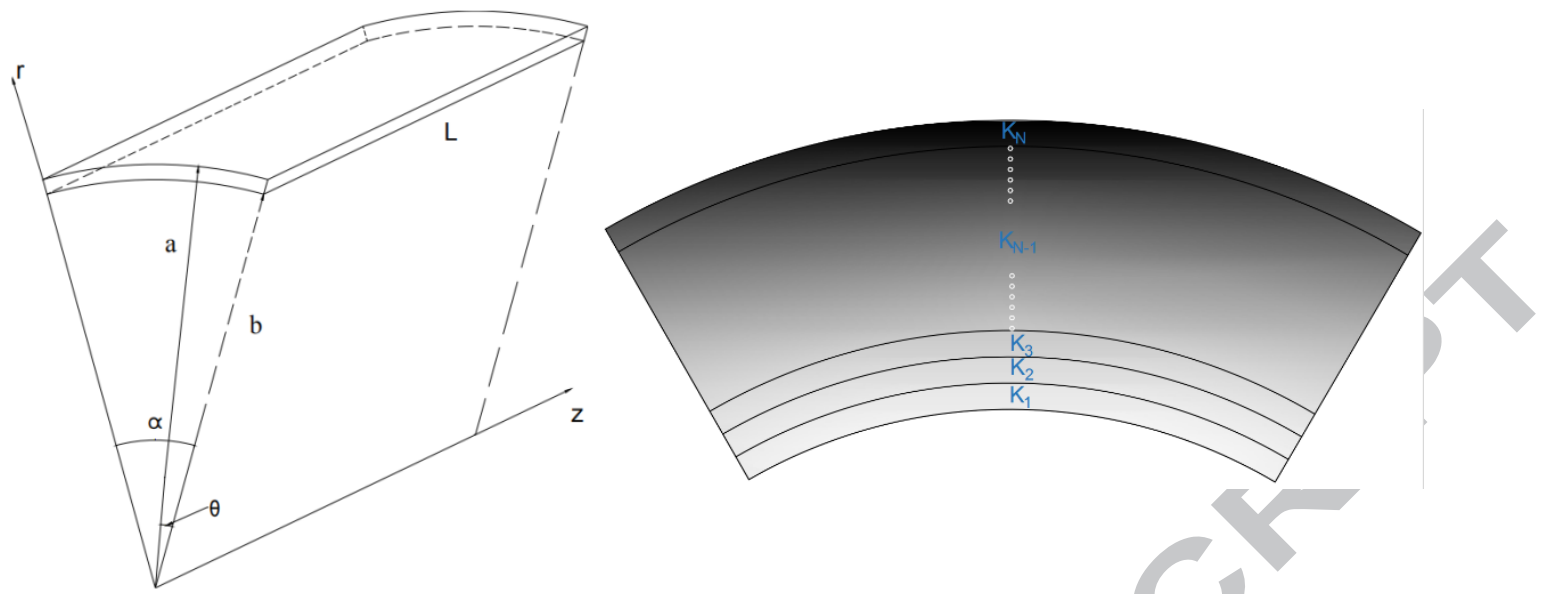

Fig. 8 The FGPM cylindrical panel and its cross section. 


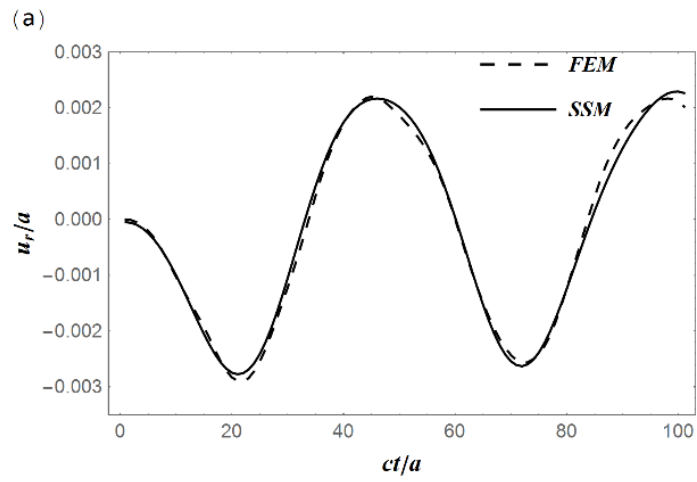

(b)

(c)

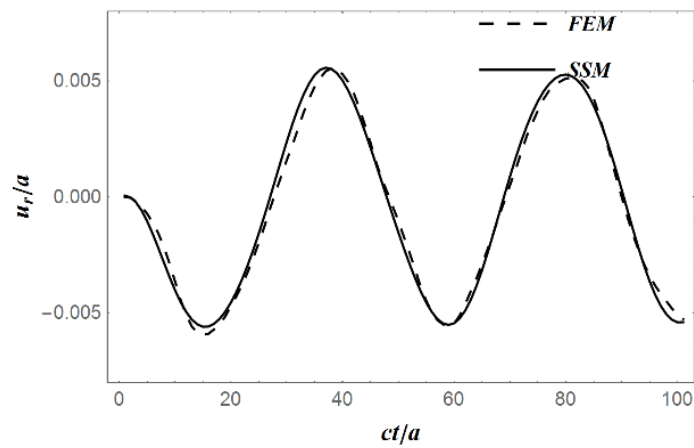

(e)

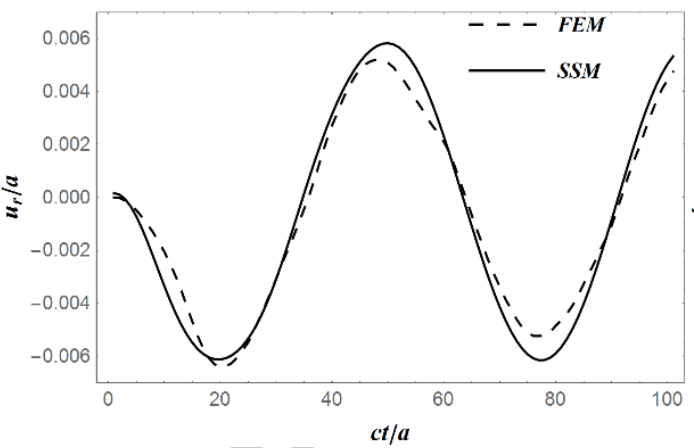

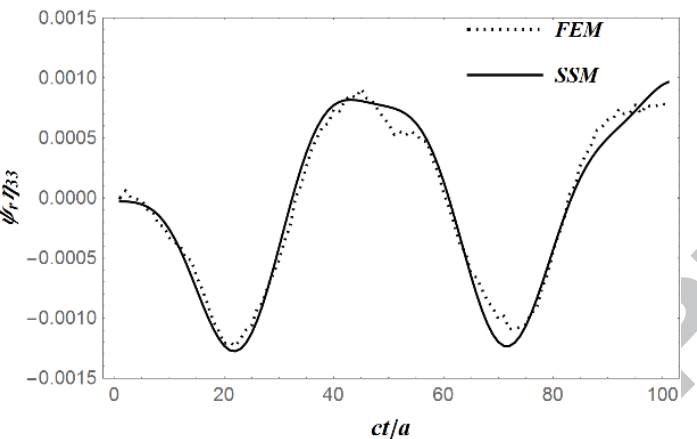

(d)

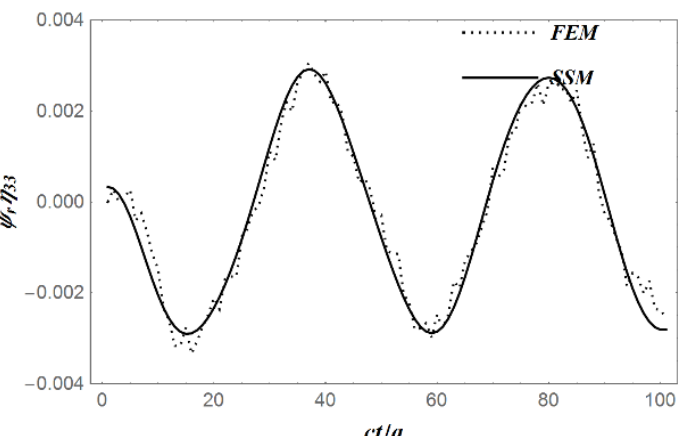

(f)

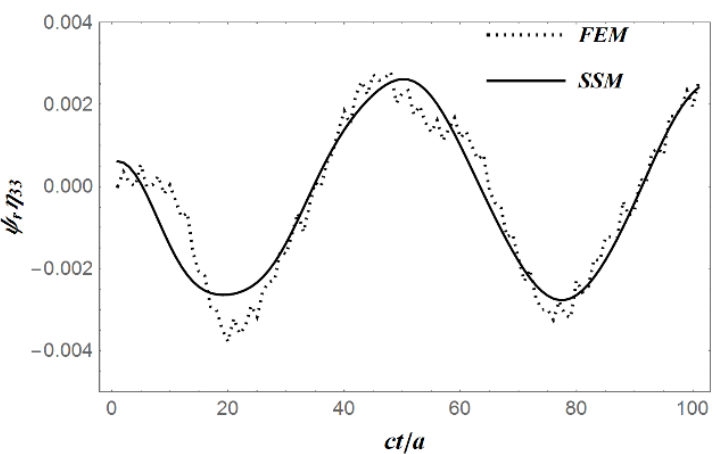

Fig. 9 Deflection and electric potential $(a, b)$ case $1 ;(c, d)$ case $2 ;(e, f)$ case 3. 
(a)

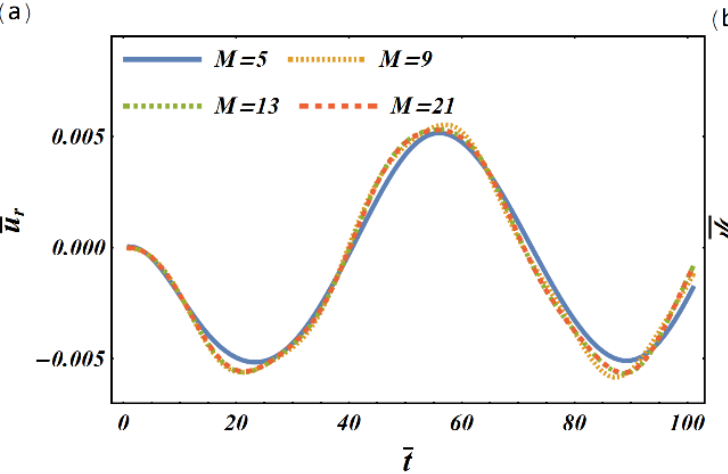

(b)

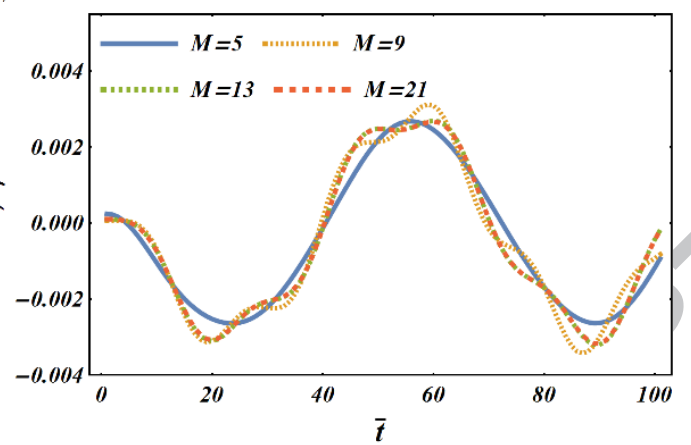

Fig. 10 Deflection and electric potential with sampling points. 


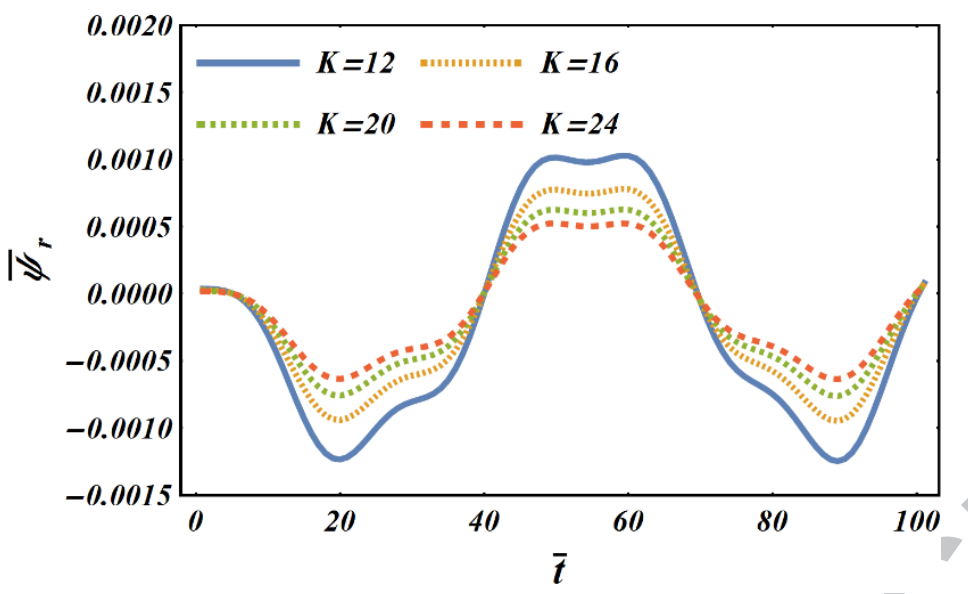

Fig. 11 Electric potential with layer numbers. 
(a)

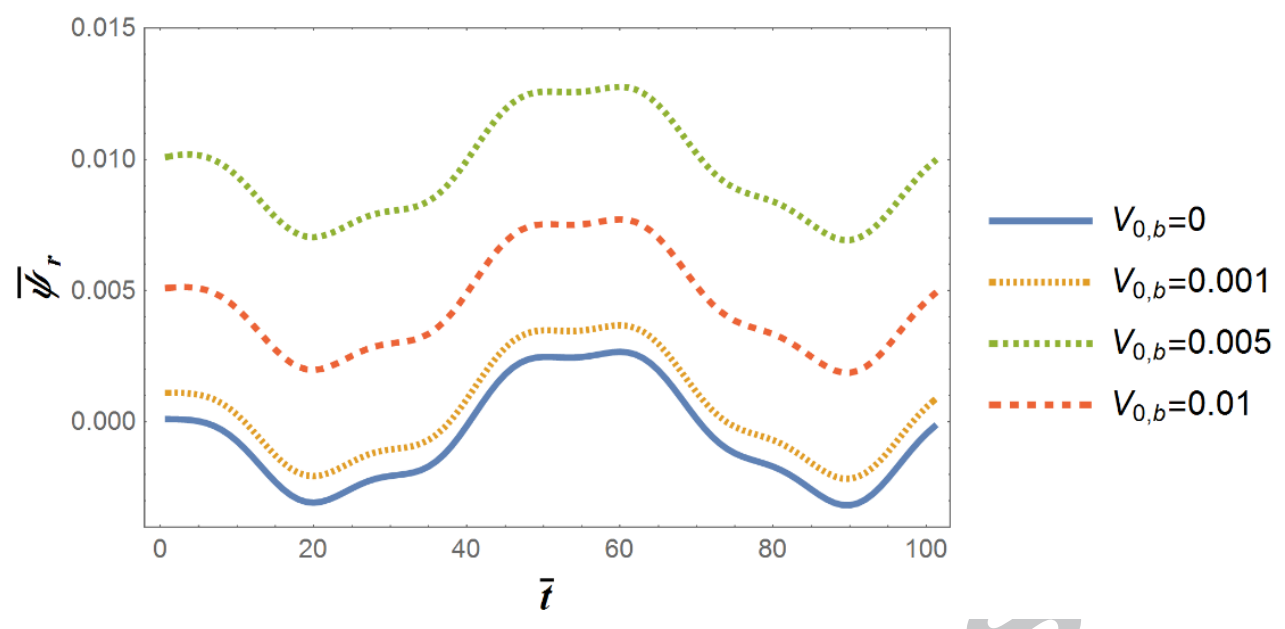

(b)

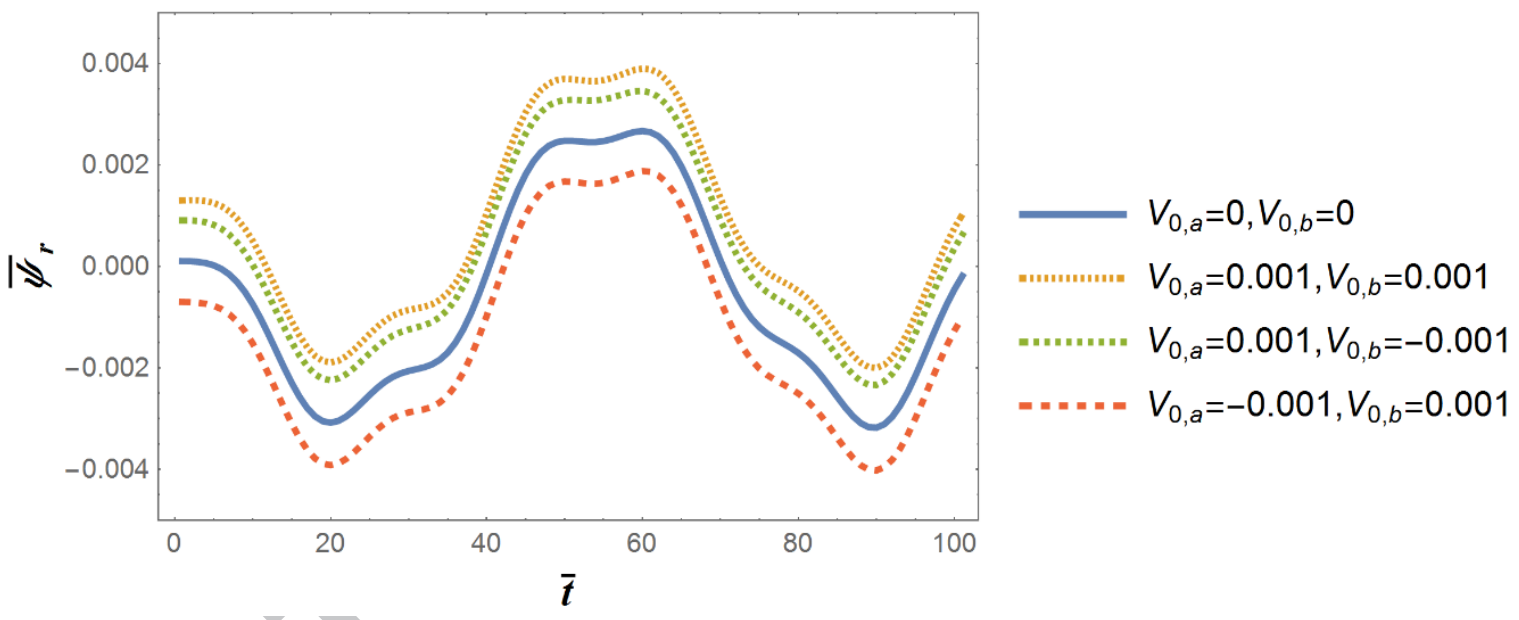

Fig. 12 Electric potential under various initial values. 

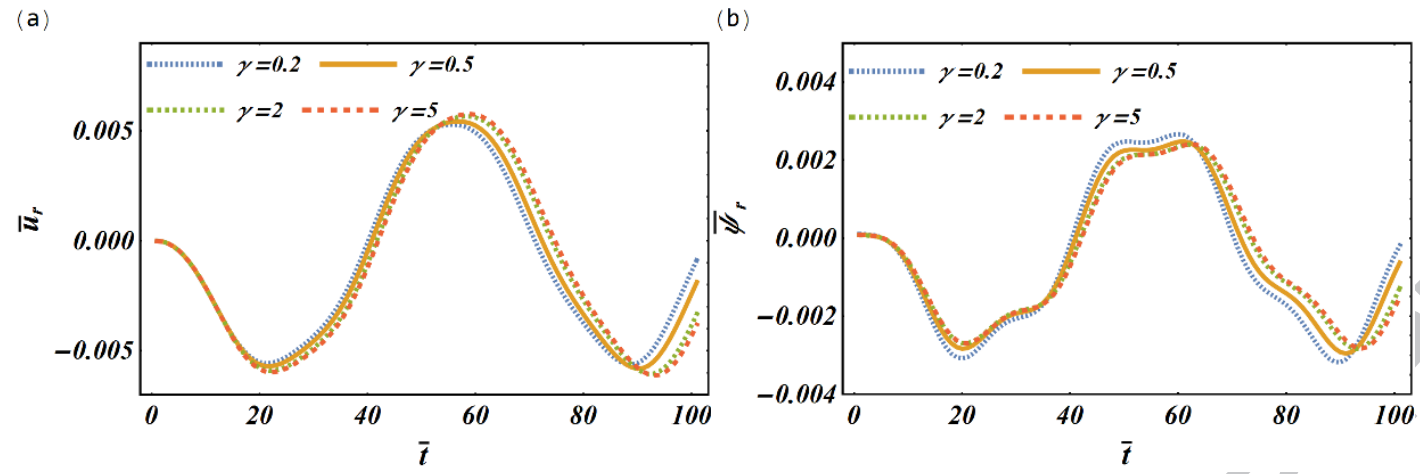

Fig. 13 Deflection and electric potential with different values of variation law. 


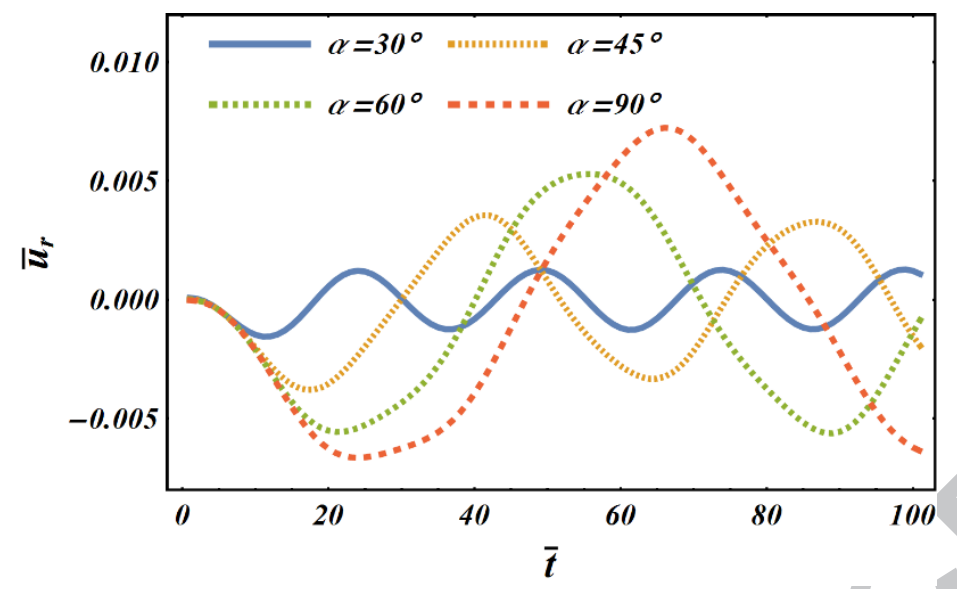

Fig. 14 Deflection history with different central angles. 